\title{
FIFA Is Right: The Penalty Shootout Should Adopt the Tennis Tiebreak Format
}

\author{
Sergio Da Silva1, Diego Mioranza², Raul Matsushita ${ }^{3}$ \\ ${ }^{1}$ Department of Economics, Federal University of Santa Catarina, Florianopolis S.C., Brazil \\ ${ }^{2}$ Catarinense Federal Institute, Blumenau S.C., Brazil \\ ${ }^{3}$ Department of Statistics, University of Brasilia, Brasilia D.F., Brazil \\ Email: professorsergiodasilva@gmail.com
}

How to cite this paper: Da Silva, S., Mioranza, D. and Matsushita, R. (2018) FIFA Is Right: The Penalty Shootout Should Adopt the Tennis Tiebreak Format. Open Access Library Journal, 5: e4427. https://doi.org/10.4236/oalib.1104427

Received: February 20, 2018

Accepted: March 12, 2018

Published: March 15, 2018

Copyright () 2018 by authors and Open Access Library Inc.

This work is licensed under the Creative

Commons Attribution International

License (CC BY 4.0).

http://creativecommons.org/licenses/by/4.0/

\begin{abstract}
In the current penalty shootout system of soccer, the team going second has to play catch-up. This possibly carries a bias in favor of the team going first. We consider this hypothesis by taking data from both soccer penalty shootouts and tennis tie breaks. We find the bias does exist in soccer, but not in tennis. This suggests the penalty shootout should adopt the tennis tiebreak format to remove the bias, as recently advocated by FIFA.
\end{abstract}

\section{Subject Areas}

Economics, Sports Science

\section{Keywords}

Soccer, Tennis, Penalty Shootout, Tennis Tiebreak, FIFA

\section{Introduction}

England's Chelsea lost the 2008 Champions League final to Manchester United on penalties when taking the shots second. Did United deserve to win? Were they just lucky? Or is the penalty shootout system bluntly unfair? FIFA's European soccer governing body UEFA is suspecting the unfair hypothesis [1] [2]. UEFA conjectures the teams taking the first penalty have a biased advantage, as they win 60 percent of shootouts. This possibly happens because the player taking the second kick is under greater mental pressure [3] [4]. On purely theoretical grounds, it can be demonstrated that the current mechanism of the soccer penalty shootout is not sequentially fair [5]-[11].

In the current penalty shootout system, teams take turns in a shootout, with the choice of who goes first decided by a coin toss. Team A goes first, then team 
$B$, then team $A$ again. This is an $A B A B$ pattern, where one side has allegedly the pressure of going second. FIFA thinks a change is needed to stop the team going second from having to play catch-up. One possible solution is to adopt the tennis tiebreak format, which follows an ABBA pattern. Team A is followed by team $B$, before team $B$ goes again. Team $A$ would then get two successive penalties, and so on until there is a winner. A coin would still be tossed to decide who goes first.

This work takes data from the penalty shootout of soccer and explicitly contrasts them with data from the tiebreak of tennis. Although FIFA claims a 60 percent advantage for the team going first, it does not show that the tiebreak of tennis is unbiased. Here, we show FIFA is right regarding soccer, and the tiebreak data do show one cannot say that the tennis system favors who goes first.

Section 2 presents the data used in the study and shows the results found, and Section 3 concludes the report.

\section{Data and Results}

We diligently collected data from the 2017 Grand Slam tennis tournaments as one of the matches progressed in order to spot 345 tiebreak situations (Table 1). We examined both men's and women's singles matches. Data from Table 1 were used to test whether a tiebreak winner is biased to the player who serves first. We expected that would not occur because of the ABBA pattern.

In contrast, we expected the first player in a soccer penalty shootout to have an advantage because of the $\mathrm{ABAB}$ pattern. To test this, we meticulously gathered data from the internet to find 232 penalty kick situations in several soccer matches from 1970 to the present day (Table 2).

As for the tiebreak, 163 players ended up as winners after serving first. This equates to 47.25 percent. However, before one can jump to the conclusion that it is better not to serve first, it can be said this result was not statistically significant. Based on a chi-square test, as a winning probability of 0.5 could not be rejected $\left(\chi^{2}=10.5, p\right.$-value $=0.306$ ), we could not conclude there was an advantage by serving first in our data sample. This result matches those in the previous literature [12] [13].

As for the penalty shootout, 138 teams ended up as winners after having the first penalty kick. This equates to 59.48 percent and, considering again the chi-square test, a winning probability of 0.5 was rejected $\left(\chi^{2}=8.35, p\right.$-value $=$ $0.004)$. This allowed us to conclude that it was significantly greater than 0.5 . If one considers $r$ to be the quantity of goals scored by a team starting the penalty shootout, and $s$ the scores of the other team, then one has 232 bivariate observations $(r, s)$ drawn from a conjoint distribution function $\operatorname{Prob}(R=r, S=s)$, where $R$ and $S$ denote the corresponding random variables (Table 3). The null hypothesis of interest is that $R$ and $S$ are exchangeable, which translates into no effect by going first. This hypothesis of exchangeability can be written as $H_{0}: \operatorname{Prob}(R \leq r, S \leq s)=\operatorname{Prob}(S \leq s, R \leq r)$, for all $(r, s)$. In addition, one can 
Table 1. Selected situations of tiebreaks for the 2017 grand slam tennis tournaments.

\begin{tabular}{|c|c|c|c|c|c|c|c|c|}
\hline & Tournament & Round & Players & & Set & $\begin{array}{l}\text { Last set game } \\
\text { winner }\end{array}$ & 1 st tiebreak serve & Tiebreak winner \\
\hline 1 & $\begin{array}{l}\text { Australian Open } \\
2017\end{array}$ & $\begin{array}{l}\text { First } \\
\text { Round }\end{array}$ & A. Murray & I. Marchenko & 2 & I. Marchenko & A. Murray & A. Murray \\
\hline 2 & $\begin{array}{l}\text { Australian Open } \\
2017\end{array}$ & $\begin{array}{l}\text { First } \\
\text { Round }\end{array}$ & A. De Minaur & G. Melzer & 4 & A. De Minaur & G. Melzer & A. De Minaur \\
\hline 3 & $\begin{array}{c}\text { Australian Open } \\
2017\end{array}$ & $\begin{array}{l}\text { First } \\
\text { Round }\end{array}$ & Q. Halys & S. Querrey & 1 & Q. Halys & S. Querrey & Q. Halys \\
\hline 4 & $\begin{array}{l}\text { Australian Open } \\
2017\end{array}$ & $\begin{array}{l}\text { First } \\
\text { Round }\end{array}$ & Q. Halys & S. Querrey & 2 & S. Querrey & Q. Halys & S. Querrey \\
\hline 5 & $\begin{array}{l}\text { Australian Open } \\
2017\end{array}$ & $\begin{array}{l}\text { First } \\
\text { Round }\end{array}$ & J. Isner & K. Kravchuk & 3 & J. Isner & K. Kravchuk & K. Kravchuk \\
\hline 6 & $\begin{array}{c}\text { Australian Open } \\
2017\end{array}$ & $\begin{array}{l}\text { First } \\
\text { Round }\end{array}$ & A. Kuznetsov & K. Nishikori & 4 & K. Nishikori & A. Kuznetsov & A. Kuznetsov \\
\hline 7 & $\begin{array}{l}\text { Australian Open } \\
2017\end{array}$ & $\begin{array}{l}\text { First } \\
\text { Round }\end{array}$ & J. Duckworth & P. Lorenzi & 2 & P. Lorenzi & J. Duckworth & P. Lorenzi \\
\hline 8 & $\begin{array}{l}\text { Australian Open } \\
2017\end{array}$ & $\begin{array}{l}\text { First } \\
\text { Round }\end{array}$ & J. Duckworth & P. Lorenzi & 3 & J. Duckworth & P. Lorenzi & J. Duckworth \\
\hline 9 & $\begin{array}{c}\text { Australian Open } \\
2017\end{array}$ & $\begin{array}{l}\text { First } \\
\text { Round }\end{array}$ & J-W. Tsonga & T. Monteiro & 3 & J-W. Tsonga & $\mathrm{J}$-W. Tsonga & T. Monteiro \\
\hline 10 & $\begin{array}{c}\text { Australian Open } \\
\quad 2017\end{array}$ & $\begin{array}{l}\text { First } \\
\text { Round }\end{array}$ & K. Khachanov & A. Mannarino & 3 & A. Mannarino & K. Khachanov & K. Khachanov \\
\hline 11 & $\begin{array}{c}\text { Australian Open } \\
2017\end{array}$ & $\begin{array}{l}\text { First } \\
\text { Round }\end{array}$ & P. Herbert & J. Sock & 2 & J. Sock & P. Herbert & J. Sock \\
\hline 12 & $\begin{array}{c}\text { Australian Open } \\
2017\end{array}$ & $\begin{array}{l}\text { First } \\
\text { Round }\end{array}$ & V. Estrella Burgos & A. Bedene & 1 & V. Estrella Burgos & A. Bedene & V. Estrella Burgos \\
\hline 13 & $\begin{array}{c}\text { Australian Open } \\
2017\end{array}$ & $\begin{array}{l}\text { First } \\
\text { Round }\end{array}$ & N. Basilashvili & P. Kohlschreiber & 3 & N. Basilashvili & P. Kohlschreiber & P. Kohlschreiber \\
\hline 14 & $\begin{array}{c}\text { Australian Open } \\
2017\end{array}$ & $\begin{array}{l}\text { First } \\
\text { Round }\end{array}$ & D. Medvedev & E. Escobedo & 3 & D. Medvedev & E. Escobedo & E. Escobedo \\
\hline 15 & $\begin{array}{c}\text { Australian Open } \\
2017\end{array}$ & $\begin{array}{c}\text { First } \\
\text { Round }\end{array}$ & J. Thompson & J. Souza & 1 & J. Thompson & J. Souza & J. Souza \\
\hline 16 & $\begin{array}{l}\text { Australian Open } \\
2017\end{array}$ & $\begin{array}{c}\text { First } \\
\text { Round }\end{array}$ & T. Haas & B. Paire & 1 & B. Paire & T. Haas & B. Paire \\
\hline 17 & $\begin{array}{c}\text { Australian Open } \\
2017\end{array}$ & $\begin{array}{c}\text { First } \\
\text { Round }\end{array}$ & D. Tursunov & R. Stepanek & 2 & R. Stepanek & D. Tursunov & R. Stepanek \\
\hline 18 & $\begin{array}{c}\text { Australian Open } \\
2017\end{array}$ & $\begin{array}{c}\text { First } \\
\text { Round }\end{array}$ & G. Dimitrov & C. Oconnel & 1 & C. Oconnel & G. Dimitrov & G. Dimitrov \\
\hline 19 & $\begin{array}{c}\text { Australian Open } \\
2017\end{array}$ & $\begin{array}{c}\text { First } \\
\text { Round }\end{array}$ & R. Albot & C. Berlocq & 2 & C. Berlocq & R. Albot & C. Berlocq \\
\hline 20 & $\begin{array}{l}\text { Australian Open } \\
2017\end{array}$ & $\begin{array}{l}\text { First } \\
\text { Round }\end{array}$ & R. Albot & C. Berlocq & 4 & R. Albot & C. Berlocq & C. Berlocq \\
\hline 21 & $\begin{array}{c}\text { Australian Open } \\
2017\end{array}$ & $\begin{array}{c}\text { First } \\
\text { Round }\end{array}$ & F. Verdasco & N. Djokovic & 2 & N. Djokovic & F. Verdasco & N. Djokovic \\
\hline 22 & $\begin{array}{c}\text { Australian Open } \\
\quad 2017\end{array}$ & $\begin{array}{l}\text { Second } \\
\text { Round }\end{array}$ & A. De Minaur & S. Querrey & 1 & S. Querrey & A. De Minaur & S. Querrey \\
\hline 23 & $\begin{array}{c}\text { Australian Open } \\
2017\end{array}$ & $\begin{array}{l}\text { Second } \\
\text { Round }\end{array}$ & J. Isner & M. Zverev & 1 & M. Zverev & J. Isner & J. Isner \\
\hline 24 & $\begin{array}{l}\text { Australian Open } \\
2017\end{array}$ & $\begin{array}{l}\text { Second } \\
\text { Round }\end{array}$ & J. Isner & M. Zverev & 2 & J. Isner & M. Zverev & J. Isner \\
\hline 25 & $\begin{array}{l}\text { Australian Open } \\
2017\end{array}$ & $\begin{array}{l}\text { Second } \\
\text { Round }\end{array}$ & J. Isner & M. Zverev & 4 & M. Zverev & J. Isner & M. Zverev \\
\hline 26 & $\begin{array}{c}\text { Australian Open } \\
2017\end{array}$ & $\begin{array}{l}\text { Second } \\
\text { Round }\end{array}$ & T. Berdych & R. Harrison & 1 & R. Harrison & T. Berdych & T. Berdych \\
\hline 27 & $\begin{array}{c}\text { Australian Open } \\
2017\end{array}$ & $\begin{array}{l}\text { Second } \\
\text { Round }\end{array}$ & N. Rubin & R. Federer & 3 & R. Federer & N. Rubin & R. Federer \\
\hline
\end{tabular}




\section{Continued}

\begin{tabular}{|c|c|c|c|c|c|c|c|c|}
\hline 28 & $\begin{array}{l}\text { Australian Open } \\
2017\end{array}$ & $\begin{array}{l}\text { Second } \\
\text { Round }\end{array}$ & P. Lorenzi & V. Troicki & 3 & P. Lorenzi & V. Troicki & V. Troicki \\
\hline 29 & $\begin{array}{c}\text { Australian Open } \\
2017\end{array}$ & $\begin{array}{l}\text { Second } \\
\text { Round }\end{array}$ & A. Seppi & N. Kyrgios & 2 & N. Kyrgios & A. Seppi & N. Kyrgios \\
\hline 30 & $\begin{array}{c}\text { Australian Open } \\
2017\end{array}$ & $\begin{array}{l}\text { Second } \\
\text { Round }\end{array}$ & B. Tomic & $\begin{array}{l}\text { V. Estrella } \\
\text { Burgos }\end{array}$ & 2 & $\begin{array}{l}\text { V. Estrella } \\
\text { Burgos }\end{array}$ & B. Tomic & B. Tomic \\
\hline 31 & $\begin{array}{c}\text { Australian Open } \\
2017\end{array}$ & $\begin{array}{l}\text { Second } \\
\text { Round }\end{array}$ & B. Tomic & $\begin{array}{l}\text { V. Estrella } \\
\text { Burgos }\end{array}$ & 4 & B. Tomic & $\begin{array}{l}\text { V. Estrella } \\
\text { Burgos }\end{array}$ & B. Tomic \\
\hline 32 & $\begin{array}{l}\text { Australian Open } \\
2017\end{array}$ & $\begin{array}{l}\text { Second } \\
\text { Round }\end{array}$ & D. Thiem & J. Thompson & 3 & J. Thompson & D. Thiem & J. Thompson \\
\hline 33 & $\begin{array}{l}\text { Australian Open } \\
2017\end{array}$ & $\begin{array}{l}\text { Second } \\
\text { Round }\end{array}$ & B. Paire & F. Fognini & 1 & B. Paire & F. Fognini & B. Paire \\
\hline 34 & $\begin{array}{l}\text { Australian Open } \\
\quad 2017\end{array}$ & $\begin{array}{l}\text { Second } \\
\text { Round }\end{array}$ & D. Istomin & N. Djokovic & 1 & D. Istomin & N. Djokovic & D. Istomin \\
\hline 35 & $\begin{array}{c}\text { Australian Open } \\
2017\end{array}$ & $\begin{array}{l}\text { Second } \\
\text { Round }\end{array}$ & D. Istomin & N. Djokovic & 4 & N. Djokovic & D. Istomin & D. Istomin \\
\hline 36 & $\begin{array}{l}\text { Australian Open } \\
2017\end{array}$ & $\begin{array}{l}\text { Third } \\
\text { Round }\end{array}$ & S. Wawrinka & V. Troicki & 4 & V. Troicki & V. Troicki & S. Wawrinka \\
\hline 37 & $\begin{array}{c}\text { Australian Open } \\
2017\end{array}$ & $\begin{array}{l}\text { Third } \\
\text { Round }\end{array}$ & S. Darcis & A. Seppi & 3 & S. Darcis & A. Seppi & A. Seppi \\
\hline 38 & $\begin{array}{c}\text { Australian Open } \\
2017\end{array}$ & $\begin{array}{l}\text { Third } \\
\text { Round }\end{array}$ & S. Darcis & A. Seppi & 4 & A. Seppi & S. Darcis & A. Seppi \\
\hline 39 & $\begin{array}{l}\text { Australian Open } \\
\quad 2017\end{array}$ & $\begin{array}{l}\text { Third } \\
\text { Round }\end{array}$ & J-W. Tsonga & J. Sock & 1 & J. Sock & J-W. Tsonga & J-W. Tsonga \\
\hline 40 & $\begin{array}{l}\text { Australian Open } \\
\quad 2017\end{array}$ & $\begin{array}{l}\text { Third } \\
\text { Round }\end{array}$ & J-W. Tsonga & J. Sock & 3 & J-W. Tsonga & J. Sock & J. Sock \\
\hline 41 & $\begin{array}{c}\text { Australian Open } \\
2017\end{array}$ & $\begin{array}{l}\text { Third } \\
\text { Round }\end{array}$ & B. Tomic & D. Evans & 2 & D. Evans & B. Tomic & D. Evans \\
\hline 42 & $\begin{array}{l}\text { Australian Open } \\
2017\end{array}$ & $\begin{array}{l}\text { Third } \\
\text { Round }\end{array}$ & B. Tomic & D. Evans & 3 & B. Tomic & D. Evans & D. Evans \\
\hline 43 & $\begin{array}{l}\text { Australian Open } \\
2017\end{array}$ & $\begin{array}{l}\text { Third } \\
\text { Round }\end{array}$ & G. Monfils & P. Kohlschreiber & 2 & G. Monfils & P. Kohlschreiber & G. Monfils \\
\hline 44 & $\begin{array}{c}\text { Australian Open } \\
2017\end{array}$ & $\begin{array}{l}\text { Third } \\
\text { Round }\end{array}$ & A. Zverev & R. Nadal & 3 & R. Nadal & A. Zverev & A. Zverev \\
\hline 45 & $\begin{array}{c}\text { Australian Open } \\
2017\end{array}$ & $\begin{array}{l}\text { Third } \\
\text { Round }\end{array}$ & R. Bautista Agut & D. Ferrer & 2 & R. Bautista Agut & D. Ferrer & D. Ferrer \\
\hline 46 & $\begin{array}{c}\text { Australian Open } \\
2017\end{array}$ & $\begin{array}{l}\text { Third } \\
\text { Round }\end{array}$ & R. Bautista Agut & D. Ferrer & 3 & D. Ferrer & R. Bautista Agut & R. Bautista Agut \\
\hline 47 & $\begin{array}{l}\text { Australian Open } \\
2017\end{array}$ & $\begin{array}{l}\text { Third } \\
\text { Round }\end{array}$ & G. Simon & M. Raonic & 2 & G. Simon & M. Raonic & M. Raonic \\
\hline 48 & $\begin{array}{l}\text { Australian Open } \\
\quad 2017\end{array}$ & $\begin{array}{l}\text { Fourth } \\
\text { Round }\end{array}$ & R. Federer & K. Nishikori & 1 & K. Nishikori & R. Federer & K. Nishikori \\
\hline 49 & $\begin{array}{l}\text { Australian Open } \\
\quad 2017\end{array}$ & $\begin{array}{l}\text { Fourth } \\
\text { Round }\end{array}$ & S. Wawrinka & A. Seppi & 1 & A. Seppi & S. Wawrinka & S. Wawrinka \\
\hline 50 & $\begin{array}{l}\text { Australian Open } \\
\quad 2017\end{array}$ & $\begin{array}{l}\text { Fourth } \\
\text { Round }\end{array}$ & S. Wawrinka & A. Seppi & 2 & S. Wawrinka & A. Seppi & S. Wawrinka \\
\hline 51 & $\begin{array}{c}\text { Australian Open } \\
2017\end{array}$ & $\begin{array}{l}\text { Fourth } \\
\text { Round }\end{array}$ & S. Wawrinka & A. Seppi & 3 & S. Wawrinka & S. Wawrinka & S. Wawrinka \\
\hline 52 & $\begin{array}{c}\text { Australian Open } \\
2017\end{array}$ & $\begin{array}{l}\text { Fourth } \\
\text { Round }\end{array}$ & J-W. Tsonga & D. Evans & 1 & D. Evans & J-W. Tsonga & D. Evans \\
\hline 53 & $\begin{array}{l}\text { Australian Open } \\
2017\end{array}$ & $\begin{array}{l}\text { Fourth } \\
\text { Round }\end{array}$ & R. Bautista Agut & M. Raonic & 1 & R. Bautista Agut & M. Raonic & M. Raonic \\
\hline 54 & $\begin{array}{l}\text { Australian Open } \\
\quad 2017\end{array}$ & $\begin{array}{l}\text { Fourth } \\
\text { Round }\end{array}$ & D. Thiem & D. Goffin & 2 & D. Thiem & D. Goffin & D. Goffin \\
\hline 55 & $\begin{array}{c}\text { Australian Open } \\
2017\end{array}$ & $\begin{array}{l}\text { Fourth } \\
\text { Round }\end{array}$ & G. Dimitrov & D. Istomin & 2 & G. Dimitrov & D. Istomin & G. Dimitrov \\
\hline
\end{tabular}




\section{Continued}

\begin{tabular}{|c|c|c|c|c|c|c|c|c|}
\hline 56 & $\begin{array}{c}\text { Australian Open } \\
2017\end{array}$ & $\begin{array}{l}\text { Quarter } \\
\text { Finals }\end{array}$ & S. Wawrinka & J-W. Tsonga & 1 & S. Wawrinka & J-W. Tsonga & S. Wawrinka \\
\hline 57 & $\begin{array}{l}\text { Australian Open } \\
2017\end{array}$ & $\begin{array}{c}\text { Quarter } \\
\text { Finals }\end{array}$ & R. Nadal & M. Raonic & 2 & R. Nadal & M. Raonic & R. Nadal \\
\hline 58 & $\begin{array}{c}\text { Australian Open } \\
\qquad 2017\end{array}$ & $\begin{array}{c}\text { Semi } \\
\text { Finals }\end{array}$ & R. Nadal & G. Dimitrov & 3 & R. Nadal & G. Dimitrov & R. Nadal \\
\hline 59 & $\begin{array}{l}\text { Australian Open } \\
\qquad 2017\end{array}$ & $\begin{array}{l}\text { Semi } \\
\text { Finals }\end{array}$ & R. Nadal & G. Dimitrov & 4 & G. Dimitrov & R. Nadal & G. Dimitrov \\
\hline 60 & $\begin{array}{c}\text { Australian Open } \\
2017\end{array}$ & $\begin{array}{l}\text { First } \\
\text { Round }\end{array}$ & E. Hozumi & C. Witthoeft & 2 & E. Hozumi & C. Witthoeft & C. Witthoeft \\
\hline 61 & $\begin{array}{l}\text { Australian Open } \\
2017\end{array}$ & $\begin{array}{l}\text { First } \\
\text { Round }\end{array}$ & D. Kasatkina & S. Peng & 2 & S. Peng & S. Peng & S. Peng \\
\hline 62 & $\begin{array}{l}\text { Australian Open } \\
2017\end{array}$ & $\begin{array}{c}\text { First } \\
\text { Round }\end{array}$ & C. Vandeweghe & R. Vinci & 2 & R. Vinci & C. Vandeweghe & C. Vandeweghe \\
\hline 63 & $\begin{array}{l}\text { Australian Open } \\
2017\end{array}$ & $\begin{array}{l}\text { First } \\
\text { Round }\end{array}$ & M. Barthel & D. Aiava & 2 & M. Barthel & D. Aiava & M. Barthel \\
\hline 64 & $\begin{array}{l}\text { Australian Open } \\
2017\end{array}$ & $\begin{array}{l}\text { First } \\
\text { Round }\end{array}$ & K. Bertens & V. Lepchenko & 2 & K. Bertens & V. Lepchenko & V. Lepchenko \\
\hline 65 & $\begin{array}{l}\text { Australian Open } \\
\quad 2017\end{array}$ & $\begin{array}{l}\text { First } \\
\text { Round }\end{array}$ & K. Kozlova & V. Williams & 1 & V. Williams & V. Williams & V. Williams \\
\hline 66 & $\begin{array}{l}\text { Australian Open } \\
2017\end{array}$ & $\begin{array}{c}\text { First } \\
\text { Round }\end{array}$ & L. Arruabarrena & Y. Putintseva & 1 & L. Arruabarrena & Y. Putintseva & Y. Putintseva \\
\hline 67 & $\begin{array}{l}\text { Australian Open } \\
2017\end{array}$ & $\begin{array}{c}\text { First } \\
\text { Round }\end{array}$ & L. Arruabarrena & Y. Putintseva & 2 & Y. Putintseva & L. Arruabarrena & Y. Putintseva \\
\hline 68 & $\begin{array}{c}\text { Australian Open } \\
2017\end{array}$ & $\begin{array}{l}\text { First } \\
\text { Round }\end{array}$ & L. Kumkhum & N. Osaka & 1 & N. Osaka & L. Kumkhum & L. Kumkhum \\
\hline 69 & $\begin{array}{c}\text { Australian Open } \\
2017\end{array}$ & $\begin{array}{l}\text { First } \\
\text { Round }\end{array}$ & K. Bondarenko & C. Garcia & 1 & C. Garcia & K. Bondarenko & C. Garcia \\
\hline 70 & $\begin{array}{c}\text { Australian Open } \\
\qquad 2017\end{array}$ & $\begin{array}{l}\text { First } \\
\text { Round }\end{array}$ & T. Babos & N. Gibbs & 1 & N. Gibbs & T. Babos & N. Gibbs \\
\hline 71 & $\begin{array}{l}\text { Australian Open } \\
2017\end{array}$ & $\begin{array}{l}\text { First } \\
\text { Round }\end{array}$ & Y. Wickmayer & L. Safarova & 2 & L. Safarova & Y. Wickmayer & L. Safarova \\
\hline 72 & $\begin{array}{l}\text { Australian Open } \\
2017\end{array}$ & $\begin{array}{l}\text { Second } \\
\text { Round }\end{array}$ & A. Kerber & C. Witthoeft & 2 & A. Kerber & C. Witthoeft & C. Witthoeft \\
\hline 73 & $\begin{array}{c}\text { Australian Open } \\
2017\end{array}$ & $\begin{array}{l}\text { Second } \\
\text { Round }\end{array}$ & K. Pliskova & I. Begu & 2 & K. Pliskova & I. Begu & K. Pliskova \\
\hline 74 & $\begin{array}{c}\text { Australian Open } \\
\qquad 2017\end{array}$ & $\begin{array}{l}\text { Second } \\
\text { Round }\end{array}$ & S. Peng & E. Bouchard & 1 & E. Bouchard & S. Peng & E. Bouchard \\
\hline 75 & $\begin{array}{c}\text { Australian Open } \\
\qquad 2017\end{array}$ & $\begin{array}{l}\text { Second } \\
\text { Round }\end{array}$ & P. Parmentier & C. Vandeweghe & 2 & C. Vandeweghe & P. Parmentier & C. Vandeweghe \\
\hline 76 & $\begin{array}{l}\text { Australian Open } \\
2017\end{array}$ & $\begin{array}{l}\text { Second } \\
\text { Round }\end{array}$ & C. Suarez Navarro & S. Cirstea & 1 & C. Suarez Navarro & S. Cirstea & S. Cirstea \\
\hline 77 & $\begin{array}{l}\text { Australian Open } \\
2017\end{array}$ & $\begin{array}{l}\text { Second } \\
\text { Round }\end{array}$ & A. Riske & S. Zhang & 1 & A. Riske & S. Zhang & A. Riske \\
\hline 78 & $\begin{array}{c}\text { Australian Open } \\
2017\end{array}$ & $\begin{array}{l}\text { Second } \\
\text { Round }\end{array}$ & D. Kovinic & T. Bacsinszky & 2 & T. Bacsinszky & T. Bacsinszky & T. Bacsinszky \\
\hline 79 & $\begin{array}{c}\text { Australian Open } \\
2017\end{array}$ & $\begin{array}{l}\text { Second } \\
\text { Round }\end{array}$ & J. Brady & H. Watson & 2 & H. Watson & J. Brady & J. Brady \\
\hline 80 & $\begin{array}{c}\text { Australian Open } \\
\qquad 2017\end{array}$ & $\begin{array}{l}\text { Second } \\
\text { Round }\end{array}$ & D. Cibulkova & S. Hsieh & 2 & S. Hsieh & D. Cibulkova & D. Cibulkova \\
\hline 81 & $\begin{array}{l}\text { Australian Open } \\
\qquad 2017\end{array}$ & $\begin{array}{l}\text { Second } \\
\text { Round }\end{array}$ & O Dodin & C. Garcia & 1 & O Dodin & C. Garcia & O Dodin \\
\hline 82 & $\begin{array}{l}\text { Australian Open } \\
2017\end{array}$ & $\begin{array}{l}\text { Third } \\
\text { Round }\end{array}$ & S. Cirstea & A. Riske & 2 & S. Cirstea & S. Cirstea & S. Cirstea \\
\hline 83 & $\begin{array}{l}\text { Australian Open } \\
2017\end{array}$ & $\begin{array}{l}\text { Third } \\
\text { Round }\end{array}$ & E. Vesnina & J. Brady & 1 & E. Vesnina & J. Brady & J. Brady \\
\hline
\end{tabular}




\section{Continued}

\begin{tabular}{|c|c|c|c|c|c|c|c|c|}
\hline 84 & $\begin{array}{l}\text { Australian Open } \\
2017\end{array}$ & $\begin{array}{l}\text { Third } \\
\text { Round }\end{array}$ & D. Cibulkova & E. Makarova & 2 & E. Makarova & D. Cibulkova & D. Cibulkova \\
\hline 85 & $\begin{array}{c}\text { Australian Open } \\
\quad 2017\end{array}$ & $\begin{array}{l}\text { Quarter } \\
\text { Finals }\end{array}$ & V. Williams & $\begin{array}{c}\text { A. } \\
\text { Pavlyuchenkova }\end{array}$ & 2 & $\begin{array}{c}\text { A. } \\
\text { Pavlyuchenkova }\end{array}$ & A. Pavlyuchenkova & A. Pavlyuchenkova \\
\hline 86 & $\begin{array}{c}\text { Australian Open } \\
2017\end{array}$ & $\begin{array}{c}\text { Semi } \\
\text { Finals }\end{array}$ & C. Vandeweghe & V. Williams & 1 & C. Vandeweghe & V. Williams & C. Vandeweghe \\
\hline 87 & Roland Garros 2017 & $\begin{array}{l}\text { First } \\
\text { Round }\end{array}$ & M. Baghdatis & N. Almagro & 1 & M. Baghdatis & N. Almagro & N. Almagro \\
\hline 88 & Roland Garros 2017 & $\begin{array}{l}\text { First } \\
\text { Round }\end{array}$ & M. Baghdatis & N. Almagro & 4 & M. Baghdatis & N. Almagro & N. Almagro \\
\hline 89 & Roland Garros 2017 & $\begin{array}{l}\text { First } \\
\text { Round }\end{array}$ & J. Isner & J. Thompson & 3 & J. Isner & J. Thompson & J. Isner \\
\hline 90 & Roland Garros 2017 & $\begin{array}{l}\text { First } \\
\text { Round }\end{array}$ & K. Khachanov & N. Jarry & 3 & N. Jarry & K. Khachanov & K. Khachanov \\
\hline 91 & Roland Garros 2017 & $\begin{array}{c}\text { First } \\
\text { Round }\end{array}$ & P. Herbert & J. Donaldson & 2 & J. Donaldson & P. Herbert & P. Herbert \\
\hline 92 & Roland Garros 2017 & $\begin{array}{l}\text { First } \\
\text { Round }\end{array}$ & E. Escobedo & S. Istomin & 1 & S. Istomin & E. Escobedo & S. Istomin \\
\hline 93 & Roland Garros 2017 & $\begin{array}{l}\text { First } \\
\text { Round }\end{array}$ & R. Albot & J. Chardy & 3 & J. Chardy & R. Albot & J. Chardy \\
\hline 94 & Roland Garros 2017 & $\begin{array}{l}\text { First } \\
\text { Round }\end{array}$ & S. Wawrinka & J. Kovalik & 2 & S. Wawrinka & J. Kovalik & S. Wawrinka \\
\hline 95 & Roland Garros 2017 & $\begin{array}{c}\text { First } \\
\text { Round }\end{array}$ & T. Monteiro & A. Muller & 1 & A. Muller & A. Muller & T. Monteiro \\
\hline 96 & Roland Garros 2017 & $\begin{array}{l}\text { First } \\
\text { Round }\end{array}$ & T. Monteiro & A. Muller & 4 & A. Muller & T. Monteiro & T. Monteiro \\
\hline 97 & Roland Garros 2017 & $\begin{array}{l}\text { First } \\
\text { Round }\end{array}$ & J-W. Tsonga & R. Olivo & 3 & R. Olivo & J-W. Tsonga & J-W. Tsonga \\
\hline 98 & Roland Garros 2017 & $\begin{array}{l}\text { First } \\
\text { Round }\end{array}$ & P. Kohlschreiber & N. Kyrgios & 2 & N. Kyrgios & P. Kohlschreiber & N. Kyrgios \\
\hline 99 & Roland Garros 2017 & $\begin{array}{l}\text { First } \\
\text { Round }\end{array}$ & R. Dutra Silva & M. Youzhny & 2 & R. Dutra Silva & M. Youzhny & R. Dutra Silva \\
\hline 100 & Roland Garros 2017 & $\begin{array}{l}\text { First } \\
\text { Round }\end{array}$ & R. Dutra Silva & M. Youzhny & 4 & M. Youzhny & R. Dutra Silva & R. Dutra Silva \\
\hline 101 & Roland Garros 2017 & $\begin{array}{l}\text { First } \\
\text { Round }\end{array}$ & M. Trungelliti & Q. Halys & 2 & Q. Halys & M. Trungelliti & Q. Halys \\
\hline 102 & Roland Garros 2017 & $\begin{array}{l}\text { First } \\
\text { Round }\end{array}$ & M. Trungelliti & Q. Halys & 3 & M. Trungelliti & Q. Halys & M. Trungelliti \\
\hline 103 & Roland Garros 2017 & $\begin{array}{l}\text { First } \\
\text { Round }\end{array}$ & G. Garcia Lopez & G. Muller & 1 & G. Garcia Lopez & G. Muller & G. Garcia Lopez \\
\hline 104 & Roland Garros 2017 & $\begin{array}{l}\text { First } \\
\text { Round }\end{array}$ & G. Garcia Lopez & G. Muller & 2 & G. Muller & G. Garcia Lopez & G. Muller \\
\hline 105 & Roland Garros 2017 & $\begin{array}{l}\text { First } \\
\text { Round }\end{array}$ & V. Troicki & E. Donskoy & 1 & V. Troicki & E. Donskoy & V. Troicki \\
\hline 106 & Roland Garros 2017 & $\begin{array}{l}\text { First } \\
\text { Round }\end{array}$ & M. Bourgue & B. Coric & 1 & B. Coric & M. Bourgue & B. Coric \\
\hline 107 & Roland Garros 2017 & $\begin{array}{l}\text { First } \\
\text { Round }\end{array}$ & Y. Sugita & S. Johnson & 3 & Y. Sugita & Y. Sugita & Y. Sugita \\
\hline 108 & Roland Garros 2017 & $\begin{array}{l}\text { First } \\
\text { Round }\end{array}$ & Y. Sugita & S. Johnson & 4 & Y. Sugita & S. Johnson & Y. Sugita \\
\hline 109 & Roland Garros 2017 & $\begin{array}{l}\text { First } \\
\text { Round }\end{array}$ & I. Karlovic & S. Tsitsipas & 1 & I. Karlovic & S. Tsitsipas & I. Karlovic \\
\hline 110 & Roland Garros 2017 & $\begin{array}{l}\text { First } \\
\text { Round }\end{array}$ & L. Pouille & J. Benneteau & 1 & J. Benneteau & L. Pouille & L. Pouille \\
\hline 111 & Roland Garros 2017 & $\begin{array}{c}\text { First } \\
\text { Round }\end{array}$ & M. Copil & $\begin{array}{c}\text { A. } \\
\text { Ramos-Vinolas }\end{array}$ & 1 & $\begin{array}{c}\text { A. } \\
\text { Ramos-Vinolas }\end{array}$ & M. Copil & M. Copil \\
\hline
\end{tabular}




\section{Continued}

\begin{tabular}{|c|c|c|c|c|c|c|c|c|}
\hline 112 & $\begin{array}{c}\text { Roland Garros } \\
2017\end{array}$ & $\begin{array}{l}\text { First } \\
\text { Round }\end{array}$ & A. Rublev & D. Schwartzman & 4 & D. Schwartzman & A. Rublev & A. Rublev \\
\hline 113 & $\begin{array}{c}\text { Roland Garros } \\
2017\end{array}$ & $\begin{array}{l}\text { Second } \\
\text { Round }\end{array}$ & A. Murray & M. Klizan & 1 & M. Klizan & A. Murray & M. Klizan \\
\hline 114 & $\begin{array}{c}\text { Roland Garros } \\
2017\end{array}$ & $\begin{array}{l}\text { Second } \\
\text { Round }\end{array}$ & A. Murray & M. Klizan & 4 & A. Murray & M. Klizan & A. Murray \\
\hline 115 & $\begin{array}{c}\text { Roland Garros } \\
2017\end{array}$ & $\begin{array}{l}\text { Second } \\
\text { Round }\end{array}$ & J. Isner & P. Lorenzi & 3 & J. Isner & P. Lorenzi & J. Isner \\
\hline 116 & $\begin{array}{c}\text { Roland Garros } \\
2017\end{array}$ & $\begin{array}{l}\text { Second } \\
\text { Round }\end{array}$ & J. Isner & P. Lorenzi & 4 & P. Lorenzi & J. Isner & J. Isner \\
\hline 117 & $\begin{array}{c}\text { Roland Garros } \\
2017\end{array}$ & $\begin{array}{l}\text { Second } \\
\text { Round }\end{array}$ & J. Chardy & K. Nishikori & 3 & K. Nishikori & J. Chardy & K. Nishikori \\
\hline 118 & $\begin{array}{c}\text { Roland Garros } \\
2017\end{array}$ & $\begin{array}{l}\text { Second } \\
\text { Round }\end{array}$ & S. Wawrinka & A. Dolgopolov & 2 & A. Dolgopolov & S. Wawrinka & S. Wawrinka \\
\hline 119 & $\begin{array}{c}\text { Roland Garros } \\
2017\end{array}$ & $\begin{array}{l}\text { Third } \\
\text { Round }\end{array}$ & A. Murray & J. del Potro & 1 & J. del Potro & A. Murray & A. Murray \\
\hline 120 & $\begin{array}{c}\text { Roland Garros } \\
2017\end{array}$ & $\begin{array}{l}\text { Third } \\
\text { Round }\end{array}$ & J. Isner & K. Khachanov & 1 & K. Khachanov & J. Isner & K. Khachanov \\
\hline 121 & $\begin{array}{c}\text { Roland Garros } \\
2017\end{array}$ & $\begin{array}{l}\text { Third } \\
\text { Round }\end{array}$ & J. Isner & K. Khachanov & 3 & K. Khachanov & J. Isner & J. Isner \\
\hline 122 & $\begin{array}{c}\text { Roland Garros } \\
2017\end{array}$ & $\begin{array}{l}\text { Third } \\
\text { Round }\end{array}$ & J. Isner & K. Khachanov & 4 & J. Isner & K. Khachanov & K. Khachanov \\
\hline 123 & $\begin{array}{c}\text { Roland Garros } \\
2017\end{array}$ & $\begin{array}{l}\text { Third } \\
\text { Round }\end{array}$ & H. Chung & K. Nishikori & 3 & K. Nishikori & H. Chung & H. Chung \\
\hline 124 & $\begin{array}{c}\text { Roland Garros } \\
2017\end{array}$ & $\begin{array}{l}\text { Third } \\
\text { Round }\end{array}$ & S. Wawrinka & F. Fognini & 1 & S. Wawrinka & S. Wawrinka & S. Wawrinka \\
\hline 125 & $\begin{array}{l}\text { Roland Garros } \\
2017\end{array}$ & $\begin{array}{l}\text { Third } \\
\text { Round }\end{array}$ & R. Gasquet & G. Monfils & 1 & R. Gasquet & G. Monfils & G. Monfils \\
\hline 126 & Roland Garros 2017 & $\begin{array}{l}\text { Third } \\
\text { Round }\end{array}$ & K. Edmund & K. Anderson & 1 & K. Edmund & K. Anderson & K. Edmund \\
\hline 127 & $\begin{array}{c}\text { Roland Garros } \\
2017\end{array}$ & $\begin{array}{l}\text { Third } \\
\text { Round }\end{array}$ & K. Edmund & K. Anderson & 2 & K. Anderson & K. Edmund & K. Anderson \\
\hline 128 & $\begin{array}{c}\text { Roland Garros } \\
2017\end{array}$ & $\begin{array}{l}\text { Third } \\
\text { Round }\end{array}$ & D. Thiem & S. Johnson & 2 & D. Thiem & S. Johnson & D. Thiem \\
\hline 129 & $\begin{array}{c}\text { Roland Garros } \\
2017\end{array}$ & $\begin{array}{l}\text { Fourth } \\
\text { Round }\end{array}$ & S. Wawrinka & G. Monfils & 2 & G. Monfils & S. Wawrinka & S. Wawrinka \\
\hline 130 & $\begin{array}{c}\text { Roland Garros } \\
2017\end{array}$ & $\begin{array}{l}\text { Fourth } \\
\text { Round }\end{array}$ & M. Raonic & P. Carreno Busta & 2 & P. Carreno Busta & M. Raonic & P. Carreno Busta \\
\hline 131 & $\begin{array}{c}\text { Roland Garros } \\
2017\end{array}$ & $\begin{array}{l}\text { Fourth } \\
\text { Round }\end{array}$ & M. Raonic & P. Carreno Busta & 3 & M. Raonic & P. Carreno Busta & M. Raonic \\
\hline 132 & $\begin{array}{c}\text { Roland Garros } \\
2017\end{array}$ & $\begin{array}{l}\text { Fourth } \\
\text { Round }\end{array}$ & A. Ramos Vinolas & N. Djokovic & 1 & A. Ramos Vinolas & N. Djokovic & N. Djokovic \\
\hline 133 & $\begin{array}{c}\text { Roland Garros } \\
2017\end{array}$ & $\begin{array}{l}\text { Quarter } \\
\text { Finals }\end{array}$ & A. Murray & K. Nishikori & 3 & K. Nishikori & K. Nishikori & A. Murray \\
\hline 134 & $\begin{array}{c}\text { Roland Garros } \\
2017\end{array}$ & $\begin{array}{l}\text { Quarter } \\
\text { Finals }\end{array}$ & D. Thiem & N. Djokovic & 1 & D. Thiem & N. Djokovic & D. Thiem \\
\hline 135 & $\begin{array}{c}\text { Roland Garros } \\
2017\end{array}$ & $\begin{array}{c}\text { Semi } \\
\text { Finals }\end{array}$ & A. Murray & S. Wawrinka & 1 & A. Murray & S. Wawrinka & A. Murray \\
\hline 136 & $\begin{array}{c}\text { Roland Garros } \\
2017\end{array}$ & $\begin{array}{l}\text { Semi } \\
\text { Finals }\end{array}$ & A. Murray & S. Wawrinka & 3 & A. Murray & S. Wawrinka & S. Wawrinka \\
\hline 137 & $\begin{array}{c}\text { Roland Garros } \\
2017\end{array}$ & $\begin{array}{l}\text { First } \\
\text { Round }\end{array}$ & M. Erakovic & S. Rogers & 1 & S. Rogers & M. Erakovic & S. Rogers \\
\hline 138 & $\begin{array}{c}\text { Roland Garros } \\
2017\end{array}$ & $\begin{array}{l}\text { First } \\
\text { Round }\end{array}$ & S. Errani & M. Doi & 1 & M. Doi & S. Errani & S. Errani \\
\hline 139 & $\begin{array}{c}\text { Roland Garros } \\
2017\end{array}$ & $\begin{array}{c}\text { First } \\
\text { Round }\end{array}$ & V. Williams & Q. Wang & 2 & Q. Wang & V. Williams & V. Williams \\
\hline
\end{tabular}




\section{Continued}

\begin{tabular}{|c|c|c|c|c|c|c|c|c|}
\hline 140 & $\begin{array}{c}\text { Roland Garros } \\
2017\end{array}$ & $\begin{array}{l}\text { First } \\
\text { Round }\end{array}$ & E. Mertens & D. Gavrilova & 1 & E. Mertens & E. Mertens & E. Mertens \\
\hline 141 & $\begin{array}{c}\text { Roland Garros } \\
2017\end{array}$ & $\begin{array}{c}\text { First } \\
\text { Round }\end{array}$ & J. Konta & S. Hsieh & 2 & J. Konta & S. Hsieh & S. Hsieh \\
\hline 142 & $\begin{array}{c}\text { Roland Garros } \\
2017\end{array}$ & $\begin{array}{c}\text { First } \\
\text { Round }\end{array}$ & C. Paquet & K. Pliskova & 1 & K. Pliskova & C. Paquet & K. Pliskova \\
\hline 143 & $\begin{array}{c}\text { Roland Garros } \\
2017\end{array}$ & $\begin{array}{c}\text { First } \\
\text { Round }\end{array}$ & A. Cornet & T. Babos & 2 & T. Babos & A. Cornet & T. Babos \\
\hline 144 & $\begin{array}{c}\text { Roland Garros } \\
2017\end{array}$ & $\begin{array}{l}\text { First } \\
\text { Round }\end{array}$ & M. Duque Marino & I. Begu & 1 & I. Begu & M. Duque Marino & M. Duque Marino \\
\hline 145 & $\begin{array}{c}\text { Roland Garros } \\
2017\end{array}$ & $\begin{array}{l}\text { Second } \\
\text { Round }\end{array}$ & S. Stosur & K. Flipkens & 2 & S. Stosur & K. Flipkens & S. Stosur \\
\hline 146 & $\begin{array}{c}\text { Roland Garros } \\
2017\end{array}$ & $\begin{array}{l}\text { Second } \\
\text { Round }\end{array}$ & B. Mattek Sands & P. Kvitova & 1 & P. Kvitova & B. Mattek Sands & B. Mattek Sands \\
\hline 147 & $\begin{array}{c}\text { Roland Garros } \\
2017\end{array}$ & $\begin{array}{l}\text { Second } \\
\text { Round }\end{array}$ & B. Mattek Sands & P. Kvitova & 2 & B. Mattek Sands & P. Kvitova & B. Mattek Sands \\
\hline 148 & $\begin{array}{c}\text { Roland Garros } \\
2017\end{array}$ & $\begin{array}{l}\text { Second } \\
\text { Round }\end{array}$ & C. Bellis & K. Bertens & 2 & C. Bellis & K. Bertens & C. Bellis \\
\hline 149 & $\begin{array}{c}\text { Roland Garros } \\
2017\end{array}$ & $\begin{array}{l}\text { Second } \\
\text { Round }\end{array}$ & O. Dodin & S. Kuznetsova & 1 & O. Dodin & O. Dodin & S. Kuznetsova \\
\hline 150 & $\begin{array}{c}\text { Roland Garros } \\
2017\end{array}$ & $\begin{array}{l}\text { Second } \\
\text { Round }\end{array}$ & G. Muguruza & A. Kontaveit & 1 & G. Muguruza & A. Kontaveit & A. Kontaveit \\
\hline 151 & $\begin{array}{c}\text { Roland Garros } \\
2017\end{array}$ & $\begin{array}{l}\text { Third } \\
\text { Round }\end{array}$ & S. Zhang & S. Kuznetsova & 1 & S. Kuznetsova & S. Zhang & S. Kuznetsova \\
\hline 152 & $\begin{array}{c}\text { Roland Garros } \\
2017\end{array}$ & $\begin{array}{l}\text { Third } \\
\text { Round }\end{array}$ & V. Cepede Royg & M. Duque Marino & 2 & V. Cepede Royg & M. Duque Marino & V. Cepede Royg \\
\hline 153 & $\begin{array}{c}\text { Roland Garros } \\
2017\end{array}$ & $\begin{array}{l}\text { Fourth } \\
\text { Round }\end{array}$ & E. Svitolina & S. Halep & 2 & E. Svitolina & S. Halep & S. Halep \\
\hline 154 & $\begin{array}{c}\text { Roland Garros } \\
2017\end{array}$ & $\begin{array}{l}\text { Fourth } \\
\text { Round }\end{array}$ & C. Garcia & K. Pliskova & 1 & K. Pliskova & C. Garcia & K. Pliskova \\
\hline 155 & $\begin{array}{c}\text { Roland Garros } \\
2017\end{array}$ & $\begin{array}{l}\text { Semi } \\
\text { Finals }\end{array}$ & J. Ostapenko & T. Bacsinszky & 1 & T. Bacsinszky & T. Bacsinszky & J. Ostapenko \\
\hline 156 & $\begin{array}{c}\text { Wimbledon } \\
2017\end{array}$ & $\begin{array}{l}\text { First } \\
\text { Round }\end{array}$ & J. Souza & D. Brown & 2 & J.Souza & D. Brown & D. Brown \\
\hline 157 & $\begin{array}{l}\text { Wimbledon } \\
2017\end{array}$ & $\begin{array}{l}\text { First } \\
\text { Round }\end{array}$ & R. Dutra Silva & B. Paire & 3 & B. Paire & B. Paire & B. Paire \\
\hline 158 & $\begin{array}{l}\text { Wimbledon } \\
2017\end{array}$ & $\begin{array}{l}\text { First } \\
\text { Round }\end{array}$ & D. Shapovalov & J. Janowicz & 4 & J. Janowicz & D. Shapovalov & J. Janowicz \\
\hline 159 & $\begin{array}{l}\text { Wimbledon } \\
2017\end{array}$ & $\begin{array}{l}\text { First } \\
\text { Round }\end{array}$ & M. Jaziri & L. Pouille & 1 & M. Jaziri & L. Pouille & M. Jaziri \\
\hline 160 & $\begin{array}{l}\text { Wimbledon } \\
2017\end{array}$ & $\begin{array}{l}\text { First } \\
\text { Round }\end{array}$ & M. Jaziri & L. Pouille & 4 & L. Pouille & M. Jaziri & L. Pouille \\
\hline 161 & $\begin{array}{l}\text { Wimbledon } \\
2017\end{array}$ & $\begin{array}{c}\text { First } \\
\text { Round }\end{array}$ & C. Berlocq & N. Basilashvili & 2 & C. Berlocq & C. Berlocq & N. Basilashvili \\
\hline 162 & $\begin{array}{l}\text { Wimbledon } \\
2017\end{array}$ & $\begin{array}{l}\text { First } \\
\text { Round }\end{array}$ & T. Fabbiano & S. Querrey & 1 & T. Fabbiano & S. Querrey & S. Querrey \\
\hline 163 & $\begin{array}{l}\text { Wimbledon } \\
2017\end{array}$ & $\begin{array}{c}\text { First } \\
\text { Round }\end{array}$ & F. Verdasco & K. Anderson & 2 & F. Verdasco & K. Anderson & K. Anderson \\
\hline 164 & $\begin{array}{l}\text { Wimbledon } \\
2017\end{array}$ & $\begin{array}{l}\text { First } \\
\text { Round }\end{array}$ & F. Verdasco & K. Anderson & 3 & K. Anderson & F. Verdasco & K. Anderson \\
\hline 165 & $\begin{array}{l}\text { Wimbledon } \\
2017\end{array}$ & $\begin{array}{c}\text { First } \\
\text { Round }\end{array}$ & T. Monteiro & A. Whittington & 3 & A. Whittington & T. Monteiro & T. Monteiro \\
\hline 166 & $\begin{array}{l}\text { Wimbledon } \\
2017\end{array}$ & $\begin{array}{l}\text { First } \\
\text { Round }\end{array}$ & T. Monteiro & A. Whittington & 4 & T. Monteiro & A. Whittington & T. Monteiro \\
\hline 167 & $\begin{array}{l}\text { Wimbledon } \\
2017\end{array}$ & $\begin{array}{l}\text { First } \\
\text { Round }\end{array}$ & A. Kuznetsov & K. Khachanov & 1 & K. Khachanov & A. Kuznetsov & K. Khachanov \\
\hline
\end{tabular}




\section{Continued}

\begin{tabular}{|c|c|c|c|c|c|c|c|c|}
\hline 168 & Wimbledon 2017 & $\begin{array}{c}\text { First } \\
\text { Round }\end{array}$ & I. Karlovic & A. Bedene & 1 & I. Karlovic & A. Bedene & I. Karlovic \\
\hline 169 & Wimbledon 2017 & $\begin{array}{l}\text { First } \\
\text { Round }\end{array}$ & I. Karlovic & A. Bedene & 2 & A. Bedene & I. Karlovic & A. Bedene \\
\hline 170 & Wimbledon 2017 & $\begin{array}{l}\text { First } \\
\text { Round }\end{array}$ & I. Karlovic & A. Bedene & 3 & I. Karlovic & A. Bedene & I. Karlovic \\
\hline 171 & Wimbledon 2017 & $\begin{array}{l}\text { First } \\
\text { Round }\end{array}$ & I. Karlovic & A. Bedene & 4 & A. Bedene & I. Karlovic & A. Bedene \\
\hline 172 & Wimbledon 2017 & $\begin{array}{l}\text { First } \\
\text { Round }\end{array}$ & J. Benneteau & S. Stakhovsky & 2 & S. Stakhovsky & J. Benneteau & S. Stakhovsky \\
\hline 173 & Wimbledon 2017 & $\begin{array}{l}\text { First } \\
\text { Round }\end{array}$ & P. Gojowczyk & M. Copil & 1 & P. Gojowczyk & M. Copil & P. Gojowczyk \\
\hline 174 & Wimbledon 2017 & $\begin{array}{l}\text { First } \\
\text { Round }\end{array}$ & F. Bagnis & R. Albot & 3 & F. Bagnis & R. Albot & R. Albot \\
\hline 175 & Wimbledon 2017 & $\begin{array}{l}\text { First } \\
\text { Round }\end{array}$ & F. Bagnis & R. Albot & 4 & R. Albot & F. Bagnis & R. Albot \\
\hline 176 & Wimbledon 2017 & $\begin{array}{l}\text { First } \\
\text { Round }\end{array}$ & M. Raonic & J. Struff & 1 & J. Struff & M. Raonic & M. Raonic \\
\hline 177 & Wimbledon 2017 & $\begin{array}{l}\text { First } \\
\text { Round }\end{array}$ & M. Raonic & J. Struff & 3 & M. Raonic & J. Struff & M. Raonic \\
\hline 178 & Wimbledon 2017 & $\begin{array}{l}\text { First } \\
\text { Round }\end{array}$ & A. Rublev & S. Travaglia & 1 & A. Rublev & S. Travaglia & S. Travaglia \\
\hline 179 & Wimbledon 2017 & $\begin{array}{l}\text { First } \\
\text { Round }\end{array}$ & J. Thompson & A. Ramos-Vinolas & 3 & A. Ramos-Vinolas & J. Thompson & A. Ramos-Vinolas \\
\hline 180 & Wimbledon 2017 & $\begin{array}{l}\text { First } \\
\text { Round }\end{array}$ & J. Sock & C. Garin & 3 & J. Sock & C. Garin & J. Sock \\
\hline 181 & Wimbledon 2017 & $\begin{array}{l}\text { First } \\
\text { Round }\end{array}$ & R. Haase & F. Tiafoe & 3 & R. Haase & F. Tiafoe & F. Tiafoe \\
\hline 182 & Wimbledon 2017 & $\begin{array}{l}\text { First } \\
\text { Round }\end{array}$ & E. Donskoy & A. Zverev & 2 & A. Zverev & E. Donskoy & A. Zverev \\
\hline 183 & Wimbledon 2017 & $\begin{array}{l}\text { First } \\
\text { Round }\end{array}$ & G. Dimitrov & D. Schwartzman & 1 & G. Dimitrov & G. Dimitrov & G. Dimitrov \\
\hline 184 & Wimbledon 2017 & $\begin{array}{l}\text { First } \\
\text { Round }\end{array}$ & T. Fritz & J. Isner & 2 & J. Isner & T. Fritz & J. Isner \\
\hline 185 & Wimbledon 2017 & $\begin{array}{l}\text { First } \\
\text { Round }\end{array}$ & M. Kukushkin & T. Daniel & 3 & T. Daniel & M. Kukushkin & M. Kukushkin \\
\hline 186 & Wimbledon 2017 & $\begin{array}{c}\text { First } \\
\text { Round }\end{array}$ & G. Simon & N. Jarry & 1 & G. Simon & N. Jarry & G. Simon \\
\hline 187 & Wimbledon 2017 & $\begin{array}{l}\text { First } \\
\text { Round }\end{array}$ & H. Zeballos & P. Lorenzi & 1 & P. Lorenzi & H. Zeballos & P. Lorenzi \\
\hline 188 & Wimbledon 2017 & $\begin{array}{c}\text { First } \\
\text { Round }\end{array}$ & H. Zeballos & P. Lorenzi & 3 & H. Zeballos & P. Lorenzi & P. Lorenzi \\
\hline 189 & Wimbledon 2017 & $\begin{array}{l}\text { First } \\
\text { Round }\end{array}$ & B. Coric & R. Harrison & 1 & B. Coric & R. Harrison & R. Harrison \\
\hline 190 & Wimbledon 2017 & $\begin{array}{l}\text { First } \\
\text { Round }\end{array}$ & B. Coric & R. Harrison & 3 & R. Harrison & B. Coric & R. Harrison \\
\hline 191 & Wimbledon 2017 & $\begin{array}{l}\text { First } \\
\text { Round }\end{array}$ & J. Chardy & T. Berdych & 3 & J. Chardy & T. Berdych & T. Berdych \\
\hline 192 & Wimbledon 2017 & $\begin{array}{l}\text { First } \\
\text { Round }\end{array}$ & Y. Sugita & B. Klein & 1 & Y. Sugita & B. Klein & Y. Sugita \\
\hline 193 & Wimbledon 2017 & $\begin{array}{l}\text { First } \\
\text { Round }\end{array}$ & J. Del Potro & T. Kokkinakis & 3 & T. Kokkinakis & J. Del Potro & J. Del Potro \\
\hline 194 & Wimbledon 2017 & $\begin{array}{l}\text { First } \\
\text { Round }\end{array}$ & A. Pavlasek & E. Escobedo & 1 & E. Escobedo & A. Pavlasek & E. Escobedo \\
\hline 195 & Wimbledon 2017 & $\begin{array}{l}\text { Second } \\
\text { Round }\end{array}$ & J. Vesely & F. Fognini & 1 & J. Vesely & F. Fognini & F. Fognini \\
\hline
\end{tabular}




\section{Continued}

\begin{tabular}{|c|c|c|c|c|c|c|c|c|}
\hline 196 & Wimbledon 2017 & $\begin{array}{l}\text { Second } \\
\text { Round }\end{array}$ & P. Herbert & B. Paire & 1 & B. Paire & P. Herbert & B. Paire \\
\hline 197 & Wimbledon 2017 & $\begin{array}{l}\text { Second } \\
\text { Round }\end{array}$ & J. Janowicz & L. Pouille & 1 & J. Janowicz & L. Pouille & J. Janowicz \\
\hline 198 & Wimbledon 2017 & $\begin{array}{l}\text { Second } \\
\text { Round }\end{array}$ & J. Janowicz & L. Pouille & 2 & L. Pouille & J. Janowicz & J. Janowicz \\
\hline 199 & Wimbledon 2017 & $\begin{array}{l}\text { Second } \\
\text { Round }\end{array}$ & K. Anderson & A. Seppi & 2 & K. Anderson & A. Seppi & K. Anderson \\
\hline 200 & Wimbledon 2017 & $\begin{array}{l}\text { Second } \\
\text { Round }\end{array}$ & T. Monteiro & K. Khachanov & 2 & T. Monteiro & K. Khachanov & K. Khachanov \\
\hline 201 & Wimbledon 2017 & $\begin{array}{l}\text { Second } \\
\text { Round }\end{array}$ & T. Monteiro & K. Khachanov & 3 & K. Khachanov & T. Monteiro & K. Khachanov \\
\hline 202 & Wimbledon 2017 & $\begin{array}{l}\text { Second } \\
\text { Round }\end{array}$ & L. Rosol & G. Muller & 2 & G. Muller & L. Rosol & L. Rosol \\
\hline 203 & Wimbledon 2017 & $\begin{array}{l}\text { Second } \\
\text { Round }\end{array}$ & K. Nishikori & S. Stakhovsky & 2 & K. Nishikori & S. Stakhovsky & S. Stakhovsky \\
\hline 204 & Wimbledon 2017 & $\begin{array}{l}\text { Second } \\
\text { Round }\end{array}$ & K. Nishikori & S. Stakhovsky & 4 & K. Nishikori & S. Stakhovsky & K. Nishikori \\
\hline 205 & Wimbledon 2017 & $\begin{array}{l}\text { Second } \\
\text { Round }\end{array}$ & F. Mayer & M. Cilic & 1 & F. Mayer & M. Cilic & M. Cilic \\
\hline 206 & Wimbledon 2017 & $\begin{array}{l}\text { Second } \\
\text { Round }\end{array}$ & M. Raonic & M. Youzhny & 2 & M. Youzhny & M. Raonic & M. Raonic \\
\hline 207 & Wimbledon 2017 & $\begin{array}{l}\text { Second } \\
\text { Round }\end{array}$ & A. Rublev & A. Ramos-Vinolas & 2 & A. Ramos-Vinolas & A. Rublev & A. Rublev \\
\hline 208 & Wimbledon 2017 & $\begin{array}{l}\text { Second } \\
\text { Round }\end{array}$ & D. Sela & J. Isner & 1 & D. Sela & J. Isner & J. Isner \\
\hline 209 & Wimbledon 2017 & $\begin{array}{l}\text { Second } \\
\text { Round }\end{array}$ & D. Sela & J. Isner & 2 & J. Isner & D. Sela & D. Sela \\
\hline 210 & Wimbledon 2017 & $\begin{array}{l}\text { Second } \\
\text { Round }\end{array}$ & D. Sela & J. Isner & 4 & D. Sela & J. Isner & D. Sela \\
\hline 211 & Wimbledon 2017 & $\begin{array}{l}\text { Second } \\
\text { Round }\end{array}$ & D. Lajovic & R. Federer & 1 & R. Federer & D. Lajovic & R. Federer \\
\hline 212 & Wimbledon 2017 & $\begin{array}{l}\text { Second } \\
\text { Round }\end{array}$ & J. Donaldson & P. Lorenzi & 2 & J. Donaldson & P. Lorenzi & J. Donaldson \\
\hline 213 & Wimbledon 2017 & $\begin{array}{l}\text { Second } \\
\text { Round }\end{array}$ & J. Donaldson & P. Lorenzi & 3 & P. Lorenzi & J. Donaldson & P. Lorenzi \\
\hline 214 & Wimbledon 2017 & $\begin{array}{l}\text { Second } \\
\text { Round }\end{array}$ & R. Harrison & T. Berdych & 3 & T. Berdych & R. Harrison & T. Berdych \\
\hline 215 & Wimbledon 2017 & $\begin{array}{l}\text { Second } \\
\text { Round }\end{array}$ & G. Monfils & K. Edmund & 1 & K. Edmund & G. Monfils & G. Monfils \\
\hline 216 & Wimbledon 2017 & $\begin{array}{l}\text { Second } \\
\text { Round }\end{array}$ & Y. Sugita & A. Mannarino & 4 & Y. Sugita & A. Mannarino & A. Mannarino \\
\hline 217 & Wimbledon 2017 & $\begin{array}{l}\text { Third } \\
\text { Round }\end{array}$ & B. Paire & J. Janowicz & 2 & B. Paire & J. Janowicz & B. Paire \\
\hline 218 & Wimbledon 2017 & $\begin{array}{l}\text { Third } \\
\text { Round }\end{array}$ & J-W. Tsonga & S. Querrey & 3 & J-W. Tsonga & S. Querrey & S. Querrey \\
\hline 219 & Wimbledon 2017 & $\begin{array}{l}\text { Third } \\
\text { Round }\end{array}$ & K. Anderson & R. Bemelmans & 1 & R. Bemelmans & K. Anderson & K. Anderson \\
\hline 220 & Wimbledon 2017 & $\begin{array}{l}\text { Third } \\
\text { Round }\end{array}$ & K. Anderson & R. Bemelmans & 3 & K. Anderson & R. Bemelmans & K. Anderson \\
\hline 221 & Wimbledon 2017 & $\begin{array}{l}\text { Third } \\
\text { Round }\end{array}$ & R. Nadal & K. Khachanov & 3 & R. Nadal & K. Khachanov & R. Nadal \\
\hline 222 & Wimbledon 2017 & $\begin{array}{l}\text { Third } \\
\text { Round }\end{array}$ & A. Bedene & G. Muller & 1 & A. Bedene & G. Muller & G. Muller \\
\hline 223 & Wimbledon 2017 & $\begin{array}{l}\text { Third } \\
\text { Round }\end{array}$ & K. Nishikori & R. Bautista Agut & 2 & K. Nishikori & R. Bautista Agut & R. Bautista Agut \\
\hline
\end{tabular}




\section{Continued}

\begin{tabular}{|c|c|c|c|c|c|c|c|c|}
\hline 224 & Wimbledon 2017 & $\begin{array}{l}\text { Third } \\
\text { Round }\end{array}$ & S. Jhonson & M. Cilic & 2 & S. Jhonson & M. Cilic & M. Cilic \\
\hline 225 & Wimbledon 2017 & $\begin{array}{l}\text { Third } \\
\text { Round }\end{array}$ & M. Raonic & A. Ramos-Vinolas & 1 & A. Ramos-Vinolas & M. Raonic & M. Raonic \\
\hline 226 & Wimbledon 2017 & $\begin{array}{l}\text { Third } \\
\text { Round }\end{array}$ & M. Zverev & R. Federer & 1 & M. Zverev & R. Federer & R. Federer \\
\hline 227 & Wimbledon 2017 & $\begin{array}{l}\text { Third } \\
\text { Round }\end{array}$ & G. Monfils & A. Mannarino & 1 & A. Mannarino & G. Monfils & A. Mannarino \\
\hline 228 & Wimbledon 2017 & $\begin{array}{l}\text { Third } \\
\text { Round }\end{array}$ & E. Gulbis & N. Djokovic & 3 & N. Djokovic & E. Gulbis & N. Djokovic \\
\hline 229 & Wimbledon 2017 & $\begin{array}{l}\text { Fourth } \\
\text { Round }\end{array}$ & A. Murray & B. Paire & 1 & B. Paire & A. Murray & A. Murray \\
\hline 230 & Wimbledon 2017 & $\begin{array}{l}\text { Fourth } \\
\text { Round }\end{array}$ & S. Querrey & K. Anderson & 2 & S. Querrey & K. Anderson & S. Querrey \\
\hline 231 & Wimbledon 2017 & $\begin{array}{l}\text { Fourth } \\
\text { Round }\end{array}$ & S. Querrey & K. Anderson & 4 & S. Querrey & K. Anderson & K. Anderson \\
\hline 232 & Wimbledon 2017 & $\begin{array}{l}\text { Fourth } \\
\text { Round }\end{array}$ & D. Thiem & T. Berdych & 2 & T. Berdych & D. Thiem & D. Thiem \\
\hline 233 & Wimbledon 2017 & $\begin{array}{l}\text { Fourth } \\
\text { Round }\end{array}$ & A. Mannarino & N. Djokovic & 2 & A. Mannarino & N. Djokovic & N. Djokovic \\
\hline 234 & Wimbledon 2017 & $\begin{array}{l}\text { Quarter } \\
\text { Finals }\end{array}$ & A. Murray & S. Querrey & 3 & A. Murray & S. Querrey & A. Murray \\
\hline 235 & Wimbledon 2017 & $\begin{array}{l}\text { Quarter } \\
\text { Finals }\end{array}$ & G. Muller & M. Cilic & 2 & M. Cilic & G. Muller & M. Cilic \\
\hline 236 & Wimbledon 2017 & $\begin{array}{l}\text { Quarter } \\
\text { Finals }\end{array}$ & M. Raonic & R. Federer & 3 & R. Federer & M. Raonic & R. Federer \\
\hline 237 & Wimbledon 2017 & $\begin{array}{l}\text { Quarter } \\
\text { Finals }\end{array}$ & T. Berdych & N. Djokovic & 1 & T. Berdych & N. Djokovic & T. Berdych \\
\hline 238 & Wimbledon 2017 & $\begin{array}{c}\text { Semi } \\
\text { Finals }\end{array}$ & S. Querrey & M. Cilic & 1 & S. Querrey & M. Cilic & S. Querrey \\
\hline 239 & Wimbledon 2017 & $\begin{array}{l}\text { Semi } \\
\text { Finals }\end{array}$ & S. Querrey & M. Cilic & 3 & M. Cilic & S. Querrey & M. Cilic \\
\hline 240 & Wimbledon 2017 & $\begin{array}{l}\text { Semi } \\
\text { Finals }\end{array}$ & R. Federer & T. Berdych & 1 & R. Federer & T. Berdych & R. Federer \\
\hline 241 & Wimbledon 2017 & $\begin{array}{l}\text { Semi } \\
\text { Finals }\end{array}$ & R. Federer & T. Berdych & 2 & T. Berdych & R. Federer & R. Federer \\
\hline 242 & Wimbledon 2017 & $\begin{array}{l}\text { First } \\
\text { Round }\end{array}$ & A. Radwanska & J. Jankovic & 1 & A. Radwanska & A. Radwanska & A. Radwanska \\
\hline 243 & Wimbledon 2017 & $\begin{array}{c}\text { First } \\
\text { Round }\end{array}$ & L.Tsurenko & J. Goerges & 1 & J. Goerges & L.Tsurenko & J. Goerges \\
\hline 244 & Wimbledon 2017 & $\begin{array}{l}\text { First } \\
\text { Round }\end{array}$ & L.Tsurenko & J. Goerges & 2 & L.Tsurenko & J. Goerges & L.Tsurenko \\
\hline 245 & Wimbledon 2017 & $\begin{array}{l}\text { First } \\
\text { Round }\end{array}$ & V. Golubic & S. Zhang & 2 & V. Golubic & V. Golubic & S. Zhang \\
\hline 246 & Wimbledon 2017 & $\begin{array}{l}\text { First } \\
\text { Round }\end{array}$ & D. Albertova & R. Ozaki & 1 & R. Ozaki & D. Albertova & D. Albertova \\
\hline 247 & Wimbledon 2017 & $\begin{array}{l}\text { First } \\
\text { Round }\end{array}$ & A. Radionova & $\begin{array}{c}\text { A. } \\
\text { Pavlyuchenkova }\end{array}$ & 2 & A. Radionova & A. Pavlyuchenkova & A. Radionova \\
\hline 248 & Wimbledon 2017 & $\begin{array}{l}\text { First } \\
\text { Round }\end{array}$ & K. Bertens & S. Cirstea & 1 & K. Bertens & S. Cirstea & S. Cirstea \\
\hline 249 & Wimbledon 2017 & $\begin{array}{c}\text { First } \\
\text { Round }\end{array}$ & S. Sorribes Tormo & N. Osaka & 2 & S. Sorribes Tormo & S. Sorribes Tormo & N. Osaka \\
\hline 250 & Wimbledon 2017 & $\begin{array}{l}\text { First } \\
\text { Round }\end{array}$ & E. Mertens & V. Williams & 1 & E. Mertens & V. Williams & V. Williams \\
\hline 251 & Wimbledon 2017 & $\begin{array}{c}\text { First } \\
\text { Round }\end{array}$ & A. Barty & E. Svitolina & 2 & A. Barty & E. Svitolina & E. Svitolina \\
\hline
\end{tabular}




\section{Continued}

\begin{tabular}{|c|c|c|c|c|c|c|c|c|}
\hline 252 & Wimbledon 2017 & $\begin{array}{c}\text { First } \\
\text { Round }\end{array}$ & D. Vekic & N. Vikhlyantseva & 1 & N. Vikhlyantseva & D. Vekic & N. Vikhlyantseva \\
\hline 253 & Wimbledon 2017 & $\begin{array}{c}\text { First } \\
\text { Round }\end{array}$ & K. Pliskova & R. Vinci & 1 & K. Pliskova & R. Vinci & K. Pliskova \\
\hline 254 & Wimbledon 2017 & $\begin{array}{c}\text { First } \\
\text { Round }\end{array}$ & M. Zanevska & H. Watson & 2 & H. Watson & M. Zanevska & H. Watson \\
\hline 255 & Wimbledon 2017 & $\begin{array}{c}\text { First } \\
\text { Round }\end{array}$ & Y. Putintseva & A. Sevastova & 2 & A. Sevastova & Y. Putintseva & A. Sevastova \\
\hline 256 & Wimbledon 2017 & $\begin{array}{c}\text { First } \\
\text { Round }\end{array}$ & M. Vondrousova & S. Peng & 1 & M. Vondrousova & S. Peng & M. Vondrousova \\
\hline 257 & Wimbledon 2017 & $\begin{array}{l}\text { Second } \\
\text { Round }\end{array}$ & S. Rogers & L. Safarova & 1 & S. Rogers & L. Safarova & L. Safarova \\
\hline 258 & Wimbledon 2017 & $\begin{array}{l}\text { Second } \\
\text { Round }\end{array}$ & S. Cirstea & B. Mattek-Sands & 2 & S. Cirstea & B. Mattek-Sands & S. Cirstea \\
\hline 259 & Wimbledon 2017 & $\begin{array}{l}\text { Second } \\
\text { Round }\end{array}$ & A. Radwanska & C. McHale & 2 & C. McHale & A. Radwanska & A. Radwanska \\
\hline 260 & Wimbledon 2017 & $\begin{array}{l}\text { Second } \\
\text { Round }\end{array}$ & V. Lepchenko & P. Hercog & 1 & P. Hercog & V. Lepchenko & V. Lepchenko \\
\hline 261 & Wimbledon 2017 & $\begin{array}{l}\text { Second } \\
\text { Round }\end{array}$ & Z. Diyas & A. Radionova & 2 & Z. Diyas & Z. Diyas & Z. Diyas \\
\hline 262 & Wimbledon 2017 & $\begin{array}{l}\text { Second } \\
\text { Round }\end{array}$ & I. Begu & A. Konjuh & 1 & I. Begu & A. Konjuh & A. Konjuh \\
\hline 263 & Wimbledon 2017 & $\begin{array}{l}\text { Second } \\
\text { Round }\end{array}$ & J. Ostapenko & F. Abanda & 2 & J. Ostapenko & F. Abanda & J. Ostapenko \\
\hline 264 & Wimbledon 2017 & $\begin{array}{l}\text { Second } \\
\text { Round }\end{array}$ & C. Giorgi & M. Keys & 2 & C. Giorgi & M. Keys & M. Keys \\
\hline 265 & Wimbledon 2017 & $\begin{array}{l}\text { Second } \\
\text { Round }\end{array}$ & C. Witthoeft & A. Sabalenka & 1 & A. Sabalenka & C. Witthoeft & C. Witthoeft \\
\hline 266 & Wimbledon 2017 & $\begin{array}{l}\text { Second } \\
\text { Round }\end{array}$ & K. Konta & D. Vekic & 1 & K. Konta & D. Vekic & K. Konta \\
\hline 267 & Wimbledon 2017 & $\begin{array}{l}\text { Second } \\
\text { Round }\end{array}$ & M. Sakkari & K. Pliskova & 1 & K. Pliskova & M. Sakkari & K. Pliskova \\
\hline 268 & Wimbledon 2017 & Third Round & A. Kerber & S. Rogers & 2 & S. Rogers & A. Kerber & A. Kerber \\
\hline 269 & Wimbledon 2017 & $\begin{array}{l}\text { Third } \\
\text { Round }\end{array}$ & P. Martic & Z. Diyas & 1 & P. Martic & Z. Diyas & P. Martic \\
\hline 270 & Wimbledon 2017 & $\begin{array}{l}\text { Third } \\
\text { Round }\end{array}$ & A. Kontaveit & C. Wozniacki & 2 & C. Wozniacki & C. Wozniacki & C. Wozniacki \\
\hline 271 & Wimbledon 2017 & $\begin{array}{l}\text { Third } \\
\text { Round }\end{array}$ & D. Cibulkova & A. Konjuh & 1 & A. Konjuh & D. Cibulkova & A. Konjuh \\
\hline 272 & Wimbledon 2017 & $\begin{array}{l}\text { Third } \\
\text { Round }\end{array}$ & N. Osaka & V. Williams & 1 & N. Osaka & V. Williams & V. Williams \\
\hline 273 & Wimbledon 2017 & $\begin{array}{l}\text { Third } \\
\text { Round }\end{array}$ & S. Peng & S. Halep & 2 & S. Halep & S. Peng & S. Halep \\
\hline 274 & Wimbledon 2017 & $\begin{array}{l}\text { Fourth } \\
\text { Round }\end{array}$ & J. Ostapenko & E. Svitolina & 2 & J. Ostapenko & J. Ostapenko & J. Ostapenko \\
\hline 275 & Wimbledon 2017 & $\begin{array}{l}\text { Fourth } \\
\text { Round }\end{array}$ & J. Konta & C. Garcia & 1 & J. Konta & C. Garcia & J. Konta \\
\hline 276 & Wimbledon 2017 & $\begin{array}{l}\text { Fourth } \\
\text { Round }\end{array}$ & V. Azarenka & S. Halep & 1 & S. Halep & V. Azarenka & S. Halep \\
\hline 277 & Wimbledon 2017 & $\begin{array}{c}\text { Quarter } \\
\text { Finals }\end{array}$ & J. Konta & S. Halep & 1 & J. Konta & S. Halep & S. Halep \\
\hline 278 & Wimbledon 2017 & $\begin{array}{c}\text { Quarter } \\
\text { Finals }\end{array}$ & J. Konta & S. Halep & 2 & S. Halep & J. Konta & J. Konta \\
\hline 279 & US Open 2017 & $\begin{array}{c}\text { First } \\
\text { Round }\end{array}$ & D. King & A. Zverev & 1 & D. King & A. Zverev & A. Zverev \\
\hline
\end{tabular}


Continued

\begin{tabular}{|c|c|c|c|c|c|c|c|c|}
\hline 280 & US Open 2017 & $\begin{array}{l}\text { First } \\
\text { Round }\end{array}$ & J. Sock & J. Thompson & 2 & J. Sock & J. Thompson & J. Thompson \\
\hline 281 & US Open 2017 & $\begin{array}{l}\text { First } \\
\text { Round }\end{array}$ & N. Almagro & S. Johnson & 2 & S. Johnson & N. Almagro & S. Johnson \\
\hline 282 & US Open 2017 & $\begin{array}{l}\text { First } \\
\text { Round }\end{array}$ & N. Almagro & S. Johnson & 3 & N. Almagro & S. Johnson & S. Johnson \\
\hline 283 & US Open 2017 & $\begin{array}{l}\text { First } \\
\text { Round }\end{array}$ & T. Kokkinakis & J. Tipsarevic & 1 & T. Kokkinakis & J. Tipsarevic & T. Kokkinakis \\
\hline 284 & US Open 2017 & $\begin{array}{c}\text { First } \\
\text { Round }\end{array}$ & T. Kokkinakis & J. Tipsarevic & 4 & J. Tipsarevic & T. Kokkinakis & J. Tipsarevic \\
\hline 285 & US Open 2017 & $\begin{array}{l}\text { First } \\
\text { Round }\end{array}$ & H. Zeballos & H. Chung & 2 & H. Zeballos & H. Chung & H. Chung \\
\hline 286 & US Open 2017 & $\begin{array}{l}\text { First } \\
\text { Round }\end{array}$ & R. Nadal & D. Lajovic & 1 & D. Lajovic & R. Nadal & R. Nadal \\
\hline 287 & US Open 2017 & $\begin{array}{c}\text { First } \\
\text { Round }\end{array}$ & R. Harrison & T. Berdych & 3 & T. Berdych & R. Harrison & T. Berdych \\
\hline 288 & US Open 2017 & $\begin{array}{c}\text { First } \\
\text { Round }\end{array}$ & J. Chardy & G. Monfils & 1 & J. Chardy & G. Monfils & G. Monfils \\
\hline 289 & US Open 2017 & $\begin{array}{l}\text { First } \\
\text { Round }\end{array}$ & A. Kuznetsov & F. Lopez & 2 & F. Lopez & A. Kuznetsov & F. Lopez \\
\hline 290 & US Open 2017 & $\begin{array}{c}\text { First } \\
\text { Round }\end{array}$ & A. Kuznetsov & F. Lopez & 3 & A. Kuznetsov & F. Lopez & A. Kuznetsov \\
\hline 291 & US Open 2017 & $\begin{array}{l}\text { First } \\
\text { Round }\end{array}$ & H. Laaksonen & J. del Potro & 2 & J. del Potro & H. Laaksonen & J. del Potro \\
\hline 292 & US Open 2017 & $\begin{array}{l}\text { First } \\
\text { Round }\end{array}$ & H. Laaksonen & J. del Potro & 3 & H. Laaksonen & J. del Potro & J. del Potro \\
\hline 293 & US Open 2017 & $\begin{array}{l}\text { Second } \\
\text { Round }\end{array}$ & Y. Sugita & L. Mayer & 1 & Y. Sugita & L. Mayer & Y. Sugita \\
\hline 294 & US Open 2017 & $\begin{array}{l}\text { Second } \\
\text { Round }\end{array}$ & S. Travaglia & V. Troicki & 1 & S. Travaglia & V. Troicki & V. Troicki \\
\hline 295 & US Open 2017 & $\begin{array}{l}\text { Second } \\
\text { Round }\end{array}$ & A. Dolgopolov & T. Berdych & 3 & A. Dolgopolov & T. Berdych & A. Dolgopolov \\
\hline 296 & US Open 2017 & $\begin{array}{l}\text { Second } \\
\text { Round }\end{array}$ & D. Goffin & G. Pella & 2 & G. Pella & D. Goffin & D. Goffin \\
\hline 297 & US Open 2017 & $\begin{array}{l}\text { Second } \\
\text { Round }\end{array}$ & D. Goffin & G. Pella & 3 & D. Goffin & G. Pella & G. Pella \\
\hline 298 & US Open 2017 & $\begin{array}{l}\text { Second } \\
\text { Round }\end{array}$ & D. Goffin & G. Pella & 4 & G. Pella & D. Goffin & D. Goffin \\
\hline 299 & US Open 2017 & $\begin{array}{l}\text { Second } \\
\text { Round }\end{array}$ & D. Young & G. Monfils & 2 & D. Young & G. Monfils & D. Young \\
\hline 300 & US Open 2017 & $\begin{array}{l}\text { Second } \\
\text { Round }\end{array}$ & A. Rublev & G. Dimitrov & 2 & A. Rublev & G. Dimitrov & A. Rublev \\
\hline 301 & US Open 2017 & $\begin{array}{l}\text { Second } \\
\text { Round }\end{array}$ & R. Federer & M. Youzhny & 2 & R. Federer & M. Youzhny & M. Youzhny \\
\hline 302 & US Open 2017 & $\begin{array}{l}\text { Second } \\
\text { Round }\end{array}$ & M. Jaziri & J. Millman & 2 & M. Jaziri & M. Jaziri & J. Millman \\
\hline 303 & US Open 2017 & $\begin{array}{l}\text { Second } \\
\text { Round }\end{array}$ & $\begin{array}{c}\text { A. } \\
\text { Mercedez-Maceiras }\end{array}$ & J. del Potro & 3 & $\begin{array}{c}\text { A. } \\
\text { Mercdez-Maceiras }\end{array}$ & J. del Potro & J. del Potro \\
\hline 304 & US Open 2017 & $\begin{array}{l}\text { Second } \\
\text { Round }\end{array}$ & A. Mannarino & B. Fratangelo & 2 & A. Mannarino & B. Fratangelo & B. Fratangelo \\
\hline 305 & US Open 2017 & $\begin{array}{l}\text { Second } \\
\text { Round }\end{array}$ & B. Coric & A. Zverev & 3 & A. Zverev & B. Coric & B. Coric \\
\hline 306 & US Open 2017 & $\begin{array}{l}\text { Second } \\
\text { Round }\end{array}$ & B. Coric & A. Zverev & 4 & B. Coric & A. Zverev & B. Coric \\
\hline 307 & US Open 2017 & $\begin{array}{l}\text { Second } \\
\text { Round }\end{array}$ & J-W. Tsonga & D. Shapovalov & 3 & D. Shapovalov & J-W. Tsonga & D. Shapovalov \\
\hline
\end{tabular}




\section{Continued}

\begin{tabular}{|c|c|c|c|c|c|c|c|c|}
\hline 308 & US Open 2017 & $\begin{array}{l}\text { Second } \\
\text { Round }\end{array}$ & S. Johnson & K. Edmund & 3 & K. Edmund & S. Johnson & K. Edmund \\
\hline 309 & US Open 2017 & $\begin{array}{l}\text { Third } \\
\text { Round }\end{array}$ & R. Nadal & L. Mayer & 1 & L. Mayer & R. Nadal & L. Mayer \\
\hline 310 & US Open 2017 & $\begin{array}{l}\text { Third } \\
\text { Round }\end{array}$ & M. Zverev & J. Isner & 3 & J. Isner & M. Zverev & M. Zverev \\
\hline 311 & US Open 2017 & $\begin{array}{l}\text { Fourth } \\
\text { Round }\end{array}$ & D. Goffin & A. Rublev & 2 & D. Goffin & A. Rublev & A. Rublev \\
\hline 312 & US Open 2017 & $\begin{array}{l}\text { Fourth } \\
\text { Round }\end{array}$ & J. Del Potro & D. Thiem & 4 & J. Del Potro & D. Thiem & J. Del Potro \\
\hline 313 & US Open 2017 & $\begin{array}{l}\text { Fourth } \\
\text { Round }\end{array}$ & P. Lorenzi & K. Anderson & 3 & K. Anderson & P. Lorenzi & P. Lorenzi \\
\hline 314 & US Open 2017 & $\begin{array}{l}\text { Fourth } \\
\text { Round }\end{array}$ & D. Shapovalov & P. Carreno Busta & 1 & P. Carreno Busta & D. Shapovalov & P. Carreno Busta \\
\hline 315 & US Open 2017 & $\begin{array}{l}\text { Fourth } \\
\text { Round }\end{array}$ & D. Shapovalov & P. Carreno Busta & 2 & D. Shapovalov & P. Carreno Busta & P. Carreno Busta \\
\hline 316 & US Open 2017 & $\begin{array}{l}\text { Fourth } \\
\text { Round }\end{array}$ & D. Shapovalov & P. Carreno Busta & 3 & P. Carreno Busta & D. Shapovalov & P. Carreno Busta \\
\hline 317 & US Open 2017 & $\begin{array}{l}\text { Fourth } \\
\text { Round }\end{array}$ & L. Pouille & D. Schwartzman & 1 & D. Schwartzman & L. Pouille & D. Schwartzman \\
\hline 318 & US Open 2017 & $\begin{array}{c}\text { Quarter } \\
\text { Finals }\end{array}$ & R. Federer & J. del Potro & 3 & R. Federer & J. del Potro & R. Federer \\
\hline 319 & US Open 2017 & $\begin{array}{c}\text { Quarter } \\
\text { Finals }\end{array}$ & S. Querrey & K. Anderson & 1 & S. Querrey & K. Anderson & K. Anderson \\
\hline 320 & US Open 2017 & $\begin{array}{c}\text { Quarter } \\
\text { Finals }\end{array}$ & S. Querrey & K. Anderson & 2 & K. Anderson & S. Querrey & S. Querrey \\
\hline 321 & US Open 2017 & $\begin{array}{l}\text { Quarter } \\
\text { Finals }\end{array}$ & S. Querrey & K. Anderson & 4 & K. Anderson & S. Querrey & K. Anderson \\
\hline 322 & US Open 2017 & $\begin{array}{l}\text { First } \\
\text { Round }\end{array}$ & A. Radwanska & P. Martic & 2 & P. Martic & A. Radwanska & A. Radwanska \\
\hline 323 & US Open 2017 & $\begin{array}{l}\text { First } \\
\text { Round }\end{array}$ & M. Vondrusova & S. Kuznetsova & 3 & S. Kuznetsova & M. Vondrusova & S. Kuznetsova \\
\hline 324 & US Open 2017 & $\begin{array}{l}\text { First } \\
\text { Round }\end{array}$ & E. Svitolina & K. Siniakova & 2 & E. Svitolina & K. Siniakova & K. Siniakova \\
\hline 325 & US Open 2017 & $\begin{array}{c}\text { First } \\
\text { Round }\end{array}$ & E. Rodina & E. Bouchard & 1 & E. Rodina & E. Bouchard & E. Rodina \\
\hline 326 & US Open 2017 & $\begin{array}{c}\text { First } \\
\text { Round }\end{array}$ & E. Mertens & M. Keys & 2 & E. Mertens & E. Mertens & M. Keys \\
\hline 327 & US Open 2017 & $\begin{array}{c}\text { First } \\
\text { Round }\end{array}$ & P. Parmentier & O. Dodin & 3 & O. Dodin & P. Parmentier & O. Dodin \\
\hline 328 & US Open 2017 & $\begin{array}{l}\text { First } \\
\text { Round }\end{array}$ & C. Liu & Y. Duan & 1 & Y. Duan & C. Liu & Y. Duan \\
\hline 329 & US Open 2017 & $\begin{array}{c}\text { First } \\
\text { Round }\end{array}$ & C. Liu & Y. Duan & 2 & Y. Duan & Y. Duan & Y. Duan \\
\hline 330 & US Open 2017 & $\begin{array}{l}\text { First } \\
\text { Round }\end{array}$ & J. Cepelova & D. Cibulkova & 1 & D. Cibulkova & J. Cepelova & J. Cepelova \\
\hline 331 & US Open 2017 & $\begin{array}{l}\text { Second } \\
\text { Round }\end{array}$ & O. Jabeur & C. Vandeweghe & 1 & O. Jabeur & C. Vandeweghe & C. Vandeweghe \\
\hline 332 & US Open 2017 & $\begin{array}{l}\text { Second } \\
\text { Round }\end{array}$ & S. Rogers & D. Gavrilova & 1 & D. Gavrilova & D. Gavrilova & S. Rogers \\
\hline 333 & US Open 2017 & $\begin{array}{l}\text { Second } \\
\text { Round }\end{array}$ & S. Rogers & D. Gavrilova & 3 & D. Gavrilova & S. Rogers & S. Rogers \\
\hline 334 & US Open 2017 & $\begin{array}{l}\text { Second } \\
\text { Round }\end{array}$ & C. Wozniacki & E. Makarova & 2 & E. Makarova & C. Wozniacki & C. Wozniacki \\
\hline 335 & US Open 2017 & $\begin{array}{l}\text { Second } \\
\text { Round }\end{array}$ & C. Suarez Navarro & M. Lucic-Baroni & 2 & M. Lucic-Baroni & C. Suarez Navarro & C. Suarez Navarro \\
\hline
\end{tabular}


Continued

\begin{tabular}{|c|c|c|c|c|c|c|c|c|}
\hline 336 & US Open 2017 & $\begin{array}{l}\text { Second } \\
\text { Round }\end{array}$ & A. Barty & A. Sasnovich & 2 & A. Barty & A. Sasnovich & A. Barty \\
\hline 337 & US Open 2017 & $\begin{array}{l}\text { Second } \\
\text { Round }\end{array}$ & S. Kenin & S. Vickery & 3 & S. Kenin & S. Kenin & S. Kenin \\
\hline 338 & US Open 2017 & $\begin{array}{l}\text { Second } \\
\text { Round }\end{array}$ & T. Babos & M. Sharapova & 1 & M. Sharapova & M. Sharapova & T. Babos \\
\hline 339 & US Open 2017 & $\begin{array}{l}\text { Third } \\
\text { Round }\end{array}$ & J. Brady & M. Niculescu & 3 & M. Niculescu & M. Niculescu & J. Brady \\
\hline 340 & US Open 2017 & $\begin{array}{l}\text { Fourth } \\
\text { Round }\end{array}$ & C. Vandeweghe & L. Safarova & 2 & L. Safarova & C. Vandeweghe & C. Vandeweghe \\
\hline 341 & US Open 2017 & $\begin{array}{l}\text { Fourth } \\
\text { Round }\end{array}$ & E. Svitolina & M. Keys & 1 & E. Svitolina & M. Keys & M. Keys \\
\hline 342 & US Open 2017 & $\begin{array}{l}\text { Fourth } \\
\text { Round }\end{array}$ & P. Kvitova & G. Muguruza & 1 & G. Muguruza & G. Muguruza & P. Kvitova \\
\hline 343 & US Open 2017 & $\begin{array}{c}\text { Quarter } \\
\text { Finals }\end{array}$ & K. Pliskova & C. Vandeweghe & 1 & C. Vandeweghe & K. Pliskova & C. Vandeweghe \\
\hline 344 & US Open 2017 & $\begin{array}{c}\text { Quarter } \\
\text { Finals }\end{array}$ & V. Williams & P. Kvitova & 3 & P. Kvitova & V. Williams & V. Williams \\
\hline 345 & US Open 2017 & $\begin{array}{c}\text { Quarter } \\
\text { Finals }\end{array}$ & S. Stephens & A. Sevastova & 3 & A. Sevastova & S. Stephens & S. Stephens \\
\hline
\end{tabular}

Table 2. Selected situations of soccer penalty kicks.

\begin{tabular}{|c|c|c|c|c|c|c|c|}
\hline & Event & Date & Sides & & Score & 1st penalty kick & Winner \\
\hline 1 & World Cup & $08 / 07 / 82$ & Germany & France & $3-3(5-4)$ & Germany & Germany \\
\hline 2 & World Cup & $21 / 06 / 86$ & Brazil & France & $1-1(3-4)$ & Brazil & France \\
\hline 3 & World Cup & $21 / 06 / 86$ & Germany & Mexico & $0-0(4-1)$ & Germany & Germany \\
\hline 4 & World Cup & $22 / 06 / 86$ & Spain & Belgium & $1-1(4-5)$ & Spain & Belgium \\
\hline 5 & World Cup & $25 / 06 / 90$ & Ireland & Romania & $0-0(5-4)$ & Ireland & Ireland \\
\hline 6 & World Cup & $30 / 06 / 90$ & Yugoslavia & Argentina & $0-0(2-3)$ & Yugoslavia & Argentina \\
\hline 7 & World Cup & $03 / 07 / 90$ & Italy & Argentina & $1-1(3-4)$ & Italy & Argentina \\
\hline 8 & World Cup & $04 / 07 / 90$ & Germany & England & $1-1(4-3)$ & Germany & Germany \\
\hline 9 & World Cup & 05/07/94 & Mexico & Bulgaria & $1-1(1-3)$ & Mexico & Bulgaria \\
\hline 10 & World Cup & $10 / 07 / 94$ & Romania & Sweden & $2-2(4-5)$ & Romania & Sweden \\
\hline 11 & World Cup & $17 / 07 / 94$ & Brazil & Italy & $0-0(3-2)$ & Italy & Brazil \\
\hline 12 & World Cup & $30 / 06 / 98$ & Argentina & England & $2-2(4-3)$ & Argentina & Argentina \\
\hline 13 & World Cup & 03/07/98 & Italy & France & $0-0(3-4)$ & Italy & France \\
\hline 14 & World Cup & 07/07/98 & Brazil & Netherlands & $1-1(4-2)$ & Brazil & Brazil \\
\hline 15 & World Cup & $16 / 06 / 02$ & Spain & Ireland & $1-1(3-2)$ & Ireland & Spain \\
\hline 16 & World Cup & $22 / 06 / 02$ & Spain & Korea & $0-0(3-5)$ & Korea & Korea \\
\hline 17 & World Cup & $26 / 06 / 06$ & Switzerland & Ukraine & $0-0(0-3)$ & Ukraine & Ukraine \\
\hline 18 & World Cup & $30 / 06 / 06$ & Germany & Argentina & $1-1(4-2)$ & Germany & Germany \\
\hline 19 & World Cup & $01 / 07 / 06$ & England & Portugal & $0-0(1-3)$ & Portugal & Portugal \\
\hline 20 & World Cup & 09/07/06 & Italy & France & $1-1(5-3)$ & Italy & Italy \\
\hline 21 & World Cup & $29 / 06 / 10$ & Paraguay & Japan & $0-0(5-3)$ & Paraguay & Paraguay \\
\hline
\end{tabular}




\section{Continued}

\begin{tabular}{|c|c|c|c|c|c|c|c|}
\hline 22 & World Cup & $02 / 07 / 10$ & Uruguay & Ghana & $1-1(4-2)$ & Uruguay & Uruguay \\
\hline 23 & World Cup & $28 / 06 / 14$ & Brazil & Chile & $1-1(3-2)$ & Brazil & Brazil \\
\hline 24 & World Cup & $29 / 06 / 14$ & Costa Rica & Greece & $1-1(5-3)$ & Costa Rica & Costa Rica \\
\hline 25 & World Cup & $05 / 07 / 14$ & Netherlands & Costa Rica & $0-0(4-3)$ & Costa Rica & Netherlands \\
\hline 26 & World Cup & $09 / 07 / 14$ & Netherlands & Argentina & $0-0(2-4)$ & Netherlands & Argentina \\
\hline 27 & Uefa Euro & $20 / 06 / 76$ & Czechoslovakia & Germany & $2-2(5-3)$ & Czechoslovakia & Czechoslovakia \\
\hline 28 & Uefa Euro & $21 / 06 / 80$ & Czechoslovakia & Italy & $1-1(9-8)$ & Italy & Czechoslovakia \\
\hline 29 & Uefa Euro & $24 / 06 / 84$ & Denmark & Spain & $1-1(5-4)$ & Denmark & Spain \\
\hline 30 & Uefa Euro & $22 / 06 / 92$ & Netherlands & Denmark & $2-2(5-4)$ & Netherlands & Denmark \\
\hline 31 & Uefa Euro & $22 / 06 / 96$ & Spain & England & $0-0(4-2)$ & England & England \\
\hline 32 & Uefa Euro & $26 / 06 / 96$ & Germany & England & $1-1(6-5)$ & England & Germany \\
\hline 33 & Uefa Euro & $26 / 06 / 96$ & France & Czech Republic & $0-0(6-5)$ & France & Czech Republic \\
\hline 34 & Uefa Euro & $29 / 06 / 00$ & Italy & Netherlands & $0-0(3-1)$ & Italy & Italy \\
\hline 35 & Uefa Euro & $24 / 06 / 04$ & Portugal & England & $2-2(6-5)$ & England & Portugal \\
\hline 36 & Uefa Euro & $26 / 06 / 04$ & Sweden & Netherlands & $0-0(5-4)$ & Sweden & Netherlands \\
\hline 37 & Uefa Euro & $20 / 06 / 08$ & Croatia & Turkey & $1-1(3-1)$ & Turkey & Turkey \\
\hline 38 & Uefa Euro & $22 / 06 / 08$ & Spain & Italy & $0-0(4-2)$ & Spain & Spain \\
\hline 39 & Uefa Euro & $24 / 06 / 12$ & England & Italy & $0-0(4-2)$ & Italy & Italy \\
\hline 40 & Uefa Euro & $27 / 06 / 12$ & Portugal & Spain & $0-0(4-2)$ & Spain & Spain \\
\hline 41 & Champions League & $04 / 11 / 70$ & Everton & Monchengladbach & $1-1(4-3)$ & Everton & Everton \\
\hline 42 & Champions League & $19 / 04 / 72$ & Celtic & Internazionale & $0-0(5-4)$ & Internazionale & Internazionale \\
\hline 43 & Champions League & 28/09/77 & Torpedo Moska & Benfica & $0-0(4-1)$ & Benfica & Benfica \\
\hline 44 & Champions League & $15 / 03 / 78$ & Juventus & Ajax & $1-1(3-0)$ & Juventus & Juventus \\
\hline 45 & Champions League & $30 / 05 / 84$ & Roma & Liverpool & $1-1(4-2)$ & Liverpool & Liverpool \\
\hline 46 & Champions League & $03 / 10 / 84$ & Berlin & Aberdeen & $2-1(5-4)$ & Aberdeen & Berlin \\
\hline 47 & Champions League & $20 / 03 / 85$ & Dnipro & Bordeaux & $1-1(5-3)$ & Bordeaux & Bordeaux \\
\hline 48 & Champions League & $16 / 04 / 86$ & Barcelona & Goteborg & $3-0(5-4)$ & Goteborg & Barcelona \\
\hline 49 & Champions League & $07 / 05 / 86$ & Steaua & Barcelona & $0-0(2-0)$ & Steaua & Steaua \\
\hline 50 & Champions League & $05 / 11 / 86$ & Juventus & Real Madrid & $1-0(3-1)$ & Real Madrid & Real Madrid \\
\hline 51 & Champions League & $25 / 05 / 88$ & PSV & Benfica & $0-0(6-5)$ & PSV & PSV \\
\hline 52 & Champions League & $05 / 10 / 88$ & Xamax & Larissa & $2-1(3-0)$ & Xamax & Xamax \\
\hline 53 & Champions League & $10 / 11 / 88$ & Crvena Zvezda & Milan & $1-1(4-2)$ & Crvena Zvezda & Milan \\
\hline 54 & Champions League & $07 / 11 / 90$ & Spartak Moska & Napoli & $0-0(5-3)$ & Spartak Moskva & Spartak Moskva \\
\hline 55 & Champions League & $07 / 11 / 90$ & Malmo & Dresden & $1-1(5-4)$ & Malmo & Dresden \\
\hline 56 & Champions League & $29 / 05 / 91$ & Crvena Zvezda & Marseille & $0-0(5-3)$ & Crvena Zvezda & Crvena Zvezda \\
\hline 57 & Champions League & $01 / 09 / 93$ & Olimpija Ljubljana & Skonto & $0-1(11-10)$ & Olimpija Ljubljana & Skonto \\
\hline 58 & Champions League & $22 / 05 / 96$ & Ajax & Juventus & $1-1(4-2)$ & Ajax & Juventus \\
\hline
\end{tabular}




\section{Continued}

\begin{tabular}{|c|c|c|c|c|c|c|c|}
\hline 59 & Champions League & $26 / 08 / 98$ & Sparta Praha & Dynamo Kyiv & $0-1(3-1)$ & Dynamo Kyiv & Dynamo Kyiv \\
\hline 60 & Champions League & $04 / 08 / 99$ & Widzew & Litex & $4-1(3-2)$ & Litex & Widzew \\
\hline 61 & Champions League & $23 / 05 / 01$ & Bayern & Valencia & $1-1(5-4)$ & Bayern & Bayern \\
\hline 62 & Champions League & $01 / 08 / 01$ & Hajduk Split & Ferencvaros & $0-0(5-4)$ & Ferencvaros & Hajduk Split \\
\hline 63 & Champions League & $28 / 08 / 02$ & Club Brugge & Shakhtar Donestsk & $1-1(4-1)$ & Club Brugge & Club Brugge \\
\hline 64 & Champions League & $28 / 05 / 03$ & Juventus & Milan & $0-0(3-2)$ & Juventus & Milan \\
\hline 65 & Champions League & $23 / 07 / 03$ & Tirana & Dinamo Tblisi & $3-0(4-2)$ & Tirana & Tirana \\
\hline 66 & Champions League & $27 / 08 / 03$ & Dortmund & Club Brugge & $2-1(4-2)$ & Dortmund & Club Brugge \\
\hline 67 & Champions League & $27 / 08 / 03$ & Newcastle & Partizan & $0-1(4-3)$ & Newcastle & Partizan \\
\hline 68 & Champions League & $13 / 04 / 05$ & PSV & Lyon & $1-1(4-2)$ & PSV & PSV \\
\hline 69 & Champions League & $25 / 05 / 05$ & Milan & Liverpool & $3-3(3-2)$ & Milan & Liverpool \\
\hline 70 & Champions League & $23 / 08 / 05$ & Partizan & Petrzalka & $0-0(4-3)$ & Petrzalka & Petrzalka \\
\hline 71 & Champions League & $24 / 08 / 05$ & Club Brugge & Valerenga & $1-0(4-3)$ & Valerenga & Club Brugge \\
\hline 72 & Champions League & 01/05/07 & Liverpool & Chelsea & $1-0(4-1)$ & Liverpool & Liverpool \\
\hline 73 & Champions League & $08 / 08 / 07$ & Slavia Praha & Zilina & $0-0(4-3)$ & Slavia Praha & Slavia Praha \\
\hline 74 & Champions League & $29 / 08 / 07$ & Celtic & Spartak Moskva & $1-1(4-3)$ & Celtic & Celtic \\
\hline 75 & Champions League & 04/03/08 & Sevilla & Fenerbahçe & $3-1(3-2)$ & Sevilla & Fenerbahçe \\
\hline 76 & Champions League & 05/03/08 & Porto & Schalke & $1-0(4-1)$ & Schalke & Schalke \\
\hline 77 & Champions League & $21 / 05 / 08$ & Manchester United & Chelsea & $1-1(6-5)$ & Manchester United & Manchester United \\
\hline 78 & Champions League & $11 / 03 / 09$ & Roma & Arsenal & $1-0(7-6)$ & Arsenal & Arsenal \\
\hline 79 & Champions League & $07 / 07 / 09$ & Sant Julia & Tre Fiori & $1-1(5-4)$ & Sant Julia & Sant Julia \\
\hline 80 & Champions League & $21 / 07 / 10$ & Lech & Inter Baki & $0-1(9-8)$ & Lech & Lech \\
\hline 81 & Champions League & $04 / 08 / 10$ & Dinamo Zagreb & Sheriff & $1-1(6-5)$ & Dinamo Zagreb & Sheriff \\
\hline 82 & Champions League & $24 / 08 / 10$ & Anderlecht & Partizan & $2-2(3-2)$ & Partizan & Partizan \\
\hline 83 & Champions League & $23 / 08 / 11$ & Genk & Haifa & $2-1(4-1)$ & Genk & Genk \\
\hline 84 & Champions League & $07 / 03 / 12$ & Apoel & Lyon & $1-0(4-3)$ & Lyon & Apoel \\
\hline 85 & Champions League & $25 / 04 / 12$ & Real Madrid & Bayern & $2-1(3-1)$ & Bayern & Bayern \\
\hline 86 & Champions League & $19 / 05 / 12$ & Bayern & Chelsea & $1-1(4-3)$ & Bayern & Chelsea \\
\hline 87 & Champions League & $10 / 07 / 12$ & B36 & Linfield & $0-0(4-3)$ & B36 & Linfield \\
\hline 88 & Champions League & $28 / 08 / 12$ & Udinese & Braga & $1-1(5-4)$ & Udinese & Braga \\
\hline 89 & Champions League & $27 / 08 / 14$ & Ludogorets & Steaua & $1-0(6-5)$ & Ludogorets & Ludogorets \\
\hline 90 & Champions League & $17 / 03 / 15$ & Atletico & Leverkusen & $1-0(3-2)$ & Atletico & Atletico \\
\hline 91 & Champions League & $15 / 03 / 16$ & Atletico & PSV & $0-0(8-7)$ & PSV & Atletico \\
\hline 92 & Champions League & $28 / 05 / 16$ & Real Madrid & Atletico & $1-1(5-3)$ & Real Madrid & Real Madrid \\
\hline 93 & Champions League & $20 / 07 / 16$ & Ferencvaros & Partizani & $1-1(3-1)$ & Partizani & Partizani \\
\hline 94 & Champions League & $03 / 08 / 16$ & Young Boys & Shakhtar Donestsk & $2-0(4-2)$ & Young Boys & Young Boys \\
\hline 95 & Copa America & $26 / 06 / 93$ & Colombia & Uruguay & $1-1(5-3)$ & Colombia & Colombia \\
\hline
\end{tabular}




\section{Continued}

\begin{tabular}{|c|c|c|c|c|c|c|c|}
\hline 96 & Copa America & $27 / 06 / 93$ & Argentina & Brazil & $1-1(6-5)$ & Brazil & Argentina \\
\hline 97 & Copa America & $01 / 07 / 93$ & Argentina & Colombia & $0-0(6-5)$ & Colombia & Argentina \\
\hline 98 & Copa America & $16 / 07 / 95$ & Colombia & Paraguay & $1-1(5-4)$ & Colombia & Colombia \\
\hline 99 & Copa America & $17 / 07 / 95$ & USA & Mexico & $0-0(4-1)$ & USA & USA \\
\hline 100 & Copa America & $17 / 07 / 95$ & Brazil & Argentina & $2-2(4-2)$ & Brazil & Brazil \\
\hline 101 & Copa America & $23 / 07 / 95$ & Uruguay & Brazil & $1-1(5-3)$ & Uruguay & Uruguay \\
\hline 102 & Copa America & $22 / 06 / 97$ & Mexico & Equador & $1-1(4-3)$ & Equador & Mexico \\
\hline 103 & Copa America & $10 / 07 / 99$ & Mexico & Peru & $3-3(4-2)$ & Mexico & Mexico \\
\hline 104 & Copa America & $10 / 07 / 99$ & Paraguay & Uruguay & $1-1(3-5)$ & Uruguay & Uruguay \\
\hline 105 & Copa America & $13 / 07 / 99$ & Uruguay & Chile & $1-1(5-3)$ & Uruguay & Uruguay \\
\hline 106 & Copa America & $28 / 07 / 01$ & Honduras & Uruguay & $2-2(5-3)$ & Honduras & Honduras \\
\hline 107 & Copa America & $21 / 07 / 04$ & Brazil & Uruguay & $1-1(5-3)$ & Brazil & Brazil \\
\hline 108 & Copa America & $25 / 07 / 04$ & Brazil & Argentina & $2-2(4-2)$ & Argentina & Brazil \\
\hline 109 & Copa America & $10 / 07 / 07$ & Brazil & Uruguay & $2-2(5-4)$ & Brazil & Brazil \\
\hline 110 & Copa America & $20 / 07 / 11$ & Paraguay & Venezuela & $0-0(5-3)$ & Paraguay & Paraguay \\
\hline 111 & Copa America & $16 / 07 / 11$ & Argentina & Uruguay & $1-1(4-5)$ & Argentina & Uruguay \\
\hline 112 & Copa America & $17 / 07 / 11$ & Brazil & Paraguay & $0-0(0-2)$ & Brazil & Paraguay \\
\hline 113 & Copa America & $27 / 06 / 15$ & Brazil & Paraguay & $1-1(3-4)$ & Brazil & Paraguay \\
\hline 114 & Copa America & $26 / 06 / 15$ & Argentina & Colombia & $0-0(5-4)$ & Colombia & Argentina \\
\hline 115 & Copa America & $04 / 07 / 15$ & Chile & Argentina & $0-0(4-1)$ & Chile & Chile \\
\hline 116 & Libertadores & $15 / 08 / 90$ & Colo Colo & Vasco & $3-3(4-5)$ & Vasco & Vasco \\
\hline 117 & Libertadores & $26 / 09 / 90$ & Olimpia & Atletico Nacional & $2-3(2-1)$ & Olimpia & Olimpia \\
\hline 118 & Libertadores & $12 / 09 / 90$ & Barcelona & River Plate & $1-0(4-3)$ & River Plate & Barcelona \\
\hline 119 & Libertadores & $17 / 06 / 92$ & Sao Paulo & Newells Old Boys & $1-0(3-2)$ & Newells Old Boys & Sao Paulo \\
\hline 120 & Libertadores & $27 / 04 / 94$ & Velez Sarsfield & Defensor & $0-0(4-3)$ & Defensor & Velez Sarsfield \\
\hline 121 & Libertadores & $17 / 08 / 94$ & Olimpia & Sao Paulo & $1-0(3-4)$ & Sao Paulo & Sao Paulo \\
\hline 122 & Libertadores & $17 / 08 / 94$ & Velez Sarsfield & Junior Barranquilla & $2-1(5-4)$ & Velez Sarsfield & Velez Sarsfield \\
\hline 123 & Libertadores & $31 / 08 / 94$ & Sao Paulo & Velez Sarsfield & $1-0(3-5)$ & Velez Sarsfield & Velez Sarsfield \\
\hline 124 & Libertadores & $16 / 08 / 95$ & River Plate & Atletico Nacional & $1-0(7-8)$ & River Plate & Atletico Nacional \\
\hline 125 & Libertadores & $14 / 04 / 97$ & Cruzeiro & El Nacional & $2-1(5-3)$ & Cruzeiro & Cruzeiro \\
\hline 126 & Libertadores & $28 / 05 / 97$ & Racing & Penarol & $1-0(3-2)$ & Racing & Racing \\
\hline 127 & Libertadores & $30 / 04 / 98$ & Olimpia & Colon & $1-0(1-2)$ & Olimpia & Colon \\
\hline 128 & Libertadores & $22 / 07 / 98$ & Cerro Porteno & Barcelona & $2-1(3-4)$ & Cerro Porteno & Barcelona \\
\hline 129 & Libertadores & $21 / 04 / 99$ & LDU & River Plate & $1-0(4-5)$ & LDU & River Plate \\
\hline 130 & Libertadores & $12 / 05 / 99$ & Corinthians & Palmeiras & $2-0(2-4)$ & Corinthians & Palmeiras \\
\hline 131 & Libertadores & $16 / 06 / 99$ & Palmeiras & Deportivo Cali & $2-1(4-3)$ & Palmeiras & Palmeiras \\
\hline 132 & Libertadores & $10 / 05 / 00$ & Bolivar & Nacional & $3-0(5-3)$ & Bolivar & Bolivar \\
\hline
\end{tabular}




\section{Continued}

\begin{tabular}{|c|c|c|c|c|c|c|c|}
\hline 133 & Libertadores & $11 / 05 / 00$ & Palmeiras & Penarol & $3-1(3-1)$ & Palmeiras & Palmeiras \\
\hline 134 & Libertadores & $09 / 05 / 00$ & Corinthians & Rosario Central & $3-2(4-3)$ & Corinthians & Corinthians \\
\hline 135 & Libertadores & $06 / 06 / 00$ & Palmeiras & Corinthians & $3-2(5-4)$ & Palmeiras & Palmeiras \\
\hline 136 & Libertadores & $21 / 06 / 00$ & Palmeiras & Boca Juniors & $0-0(2-4)$ & Palmeiras & Boca Juniors \\
\hline 137 & Libertadores & $28 / 06 / 01$ & Boca Juniors & Cruz Azul & $0-1(3-1)$ & Boca Juniors & Boca Juniors \\
\hline 138 & Libertadores & $01 / 05 / 02$ & Sao Caetano & Universidad Catolica & $1-1(4-2)$ & Sao Caetano & Sao Caetano \\
\hline 139 & Libertadores & $17 / 07 / 02$ & Gremio & Olimpia & $1-0(4-5)$ & Gremio & Olimpia \\
\hline 140 & Libertadores & $31 / 07 / 02$ & Sao Caetano & Olimpia & $1-2(2-4)$ & Sao Caetano & Olimpia \\
\hline 141 & Libertadores & $06 / 05 / 03$ & Medellin & Cerro Porteno & $0-1(4-2)$ & Medellin & Medellin \\
\hline 142 & Libertadores & $13 / 05 / 03$ & Racing & America de Cali & $0-0(5-6)$ & Racing & America de Cali \\
\hline 143 & Libertadores & $07 / 05 / 03$ & Santos & Nacional & $2-2(3-1)$ & Nacional & Santos \\
\hline 144 & Libertadores & $13 / 05 / 03$ & Deportivo Cali & Cruz Azul & $0-0(2-3)$ & Deportivo Cali & Cruz Azul \\
\hline 145 & Libertadores & $28 / 04 / 04$ & Sao Caetano & Independiente & $2-2(4-2)$ & Independiente & Sao Caetano \\
\hline 146 & Libertadores & $13 / 05 / 04$ & Once Caldas & Barcelona & $1-1(4-2)$ & Barcelona & Once Caldas \\
\hline 147 & Libertadores & $12 / 05 / 04$ & Sao Paulo & Rosario Central & $2-1(5-4)$ & Sao Paulo & Sao Paulo \\
\hline 148 & Libertadores & $13 / 05 / 04$ & Cruzeiro & Deportivo Cali & $2-1(0-3)$ & Deportivo Cali & Deportivo Cali \\
\hline 149 & Libertadores & $11 / 05 / 04$ & River Plate & Santos Laguna & $1-2(4-2)$ & River Plate & River Plate \\
\hline 150 & Libertadores & $25 / 05 / 04$ & Boca Juniors & Sao Caetano & $1-1(4-3)$ & Boca Juniors & Boca Juniors \\
\hline 151 & Libertadores & $17 / 06 / 04$ & River Plate & Boca Juniors & $2-1(4-5)$ & River Plate & Boca Juniors \\
\hline 152 & Libertadores & $01 / 07 / 04$ & Once Caldas & Boca Juniors & $1-1(2-0)$ & Once Caldas & Once Caldas \\
\hline 153 & Libertadores & $04 / 05 / 06$ & Libertad & Tigres & $0-0(5-3)$ & Libertad & Libertad \\
\hline 154 & Libertadores & $19 / 07 / 06$ & Sao Paulo & Estudiantes & $1-0(4-3)$ & Sao Paulo & Sao Paulo \\
\hline 155 & Libertadores & $23 / 05 / 07$ & Gremio & Defensor & $2-0(4-2)$ & Defensor & Gremio \\
\hline 156 & Libertadores & $22 / 05 / 08$ & LDU & San Lorenzo & $1-1(5-3)$ & LDU & LDU \\
\hline 157 & Libertadores & $02 / 07 / 08$ & Fluminense & LDU & $3-1(1-3)$ & LDU & LDU \\
\hline 158 & Libertadores & $12 / 05 / 09$ & Sport & Palmeiras & $1-0(1-3)$ & Palmeiras & Palmeiras \\
\hline 159 & Libertadores & $09 / 02 / 10$ & Universidad Catolica & Colon & $3-2(5-3)$ & Universidad Catolica & Universidad Catolica \\
\hline 160 & Libertadores & $04 / 05 / 10$ & Sao Paulo & Universitario & $0-0(3-1)$ & Universitario & Sao Paulo \\
\hline 161 & Libertadores & $05 / 05 / 11$ & Cerro Porteno & Estudiantes & $0-0(5-3)$ & Cerro Porteno & Cerro Porteno \\
\hline 162 & Libertadores & $09 / 05 / 12$ & Lanus & Vasco & $2-1(4-5)$ & Lanus & Vasco \\
\hline 163 & Libertadores & $24 / 05 / 12$ & Santos & Velez Sarsfield & $1-0(4-2)$ & Velez Sarsfield & Santos \\
\hline 164 & Libertadores & $24 / 05 / 12$ & U de Chile & Libertad & $1-1(5-3)$ & U de Chile & U de Chile \\
\hline 165 & Libertadores & $30 / 01 / 13$ & Gremio & LDU & $1-0(5-4)$ & Gremio & Gremio \\
\hline 166 & Libertadores & $09 / 05 / 13$ & Nacional & Real Garcilaso & $1-0(1-4)$ & Real Garcilaso & Real Garcilaso \\
\hline 167 & Libertadores & $29 / 05 / 13$ & Newells Old Boys & Boca Juniors & $0-0(10-9)$ & Boca Juniors & Newells Old Boys \\
\hline 168 & Libertadores & $10 / 07 / 13$ & Atletico Mineiro & Newells Old Boys & $2-0(3-2)$ & Atletico Mineiro & Atletico Mineiro \\
\hline 169 & Libertadores & $24 / 07 / 13$ & Atletico Mineiro & Olimpia & $2-0(4-3)$ & Olimpia & Atletico Mineiro \\
\hline
\end{tabular}




\section{Continued}

\begin{tabular}{|c|c|c|c|c|c|c|c|}
\hline 170 & Libertadores & $05 / 02 / 14$ & Atletico Paranaense & Sporting Cristal & $2-1(5-4)$ & Atletico Paranaense & Atletico Paranaense \\
\hline 171 & Libertadores & $29 / 04 / 14$ & Defensor & The Strongest & $2-0(4-2)$ & Defensor & Defensor \\
\hline 172 & Libertadores & $30 / 04 / 14$ & Gremio & San Lorenzo & $1-0(2-4)$ & Gremio & San Lorenzo \\
\hline 173 & Libertadores & $13 / 05 / 15$ & Cruzeiro & Sao Paulo & $1-0(4-3)$ & Sao Paulo & Cruzeiro \\
\hline 174 & Libertadores & $19 / 05 / 16$ & Boca Juniors & Nacional & $1-1(4-3)$ & Nacional & Boca Juniors \\
\hline 175 & Libertadores & $24 / 05 / 16$ & Pumas & Ind. Del Valle & $2-1(3-5)$ & Ind. Del Valle & Ind. Del Valle \\
\hline 176 & Sul Americana & $26 / 09 / 02$ & Alianza & Barcelona & $2-1(6-5)$ & Alianza & Alianza \\
\hline 177 & Sul Americana & $29 / 10 / 02$ & S. Wanderers & Atletico Nacional & $1-0(5-6)$ & Atletico Nacional & Atletico Nacional \\
\hline 178 & Sul Americana & $23 / 10 / 02$ & Racing & San Lorenzo & $2-0(3-4)$ & Racing & San Lorenzo \\
\hline 179 & Sul Americana & $12 / 11 / 02$ & Nacional & Atletico Nacional & $2-1(3-5)$ & Atletico Nacional & Atletico Nacional \\
\hline 180 & Sul Americana & $28 / 08 / 03$ & Libertad & Guarani & $0-1(4-2)$ & Guarani & Libertad \\
\hline 181 & Sul Americana & $02 / 09 / 03$ & Osorno & Catolica & $1-2(3-5)$ & Catolica & Catolica \\
\hline 182 & Sul Americana & $19 / 08 / 03$ & Bolivar & Strongest & $1-1(2-4)$ & Strongest & Strongest \\
\hline 183 & Sul Americana & $03 / 12 / 03$ & Sao Paulo & River Plate & $2-0(2-4)$ & Sao Paulo & River Plate \\
\hline 184 & Sul Americana & $22 / 09 / 04$ & Sao Paulo & Sao Caetano & $1-1(4-1)$ & Sao Paulo & Sao Paulo \\
\hline 185 & Sul Americana & $22 / 09 / 04$ & Flamengo & Santos & $2-2(4-5)$ & Flamengo & Santos \\
\hline 186 & Sul Americana & $04 / 09 / 04$ & Internacional & Figueirense & $1-1(4-2)$ & Internacional & Internacional \\
\hline 187 & Sul Americana & $29 / 09 / 04$ & Boca Juniors & San Lorenzo & $2-1(4-1)$ & Boca Juniors & Boca Juniors \\
\hline 188 & Sul Americana & $10 / 11 / 04$ & Cerro Porteno & Boca Juniors & $0-0(7-8)$ & Boca Juniors & Boca Juniors \\
\hline 189 & Sul Americana & $23 / 08 / 05$ & Atletico Nacional & Deportivo Cali & $2-0(7-6)$ & Deportivo Cali & Atletico Nacional \\
\hline 190 & Sul Americana & $23 / 08 / 05$ & Cerro Porteno & Guarani & $1-2(4-3)$ & Guarani & Cerro Porteno \\
\hline 191 & Sul Americana & $24 / 08 / 05$ & A Atletico & Universitario & $1-1(4-1)$ & A Atletico & A Atletico \\
\hline 192 & Sul Americana & $31 / 08 / 05$ & Santos & Fluminense & $2-1(2-4)$ & Fluminense & Fluminense \\
\hline 193 & Sul Americana & $18 / 12 / 05$ & Boca Juniors & Pumas & $1-1(4-3)$ & Pumas & Boca Juniors \\
\hline 194 & Sul Americana & $14 / 09 / 06$ & Fluminense & Botafogo & $1-1(4-2)$ & Botafogo & Fluminense \\
\hline 195 & Sul Americana & $13 / 09 / 06$ & Cruzeiro & Santos & $1-0(3-4)$ & Cruzeiro & Santos \\
\hline 196 & Sul Americana & $31 / 08 / 06$ & Colo Colo & Huachipato & $1-2(5-3)$ & Colo Colo & Colo Colo \\
\hline 197 & Sul Americana & $12 / 10 / 06$ & Boca Juniors & Nacional & $2-1(1-3)$ & Nacional & Nacional \\
\hline 198 & Sul Americana & $23 / 08 / 07$ & Bolognesi & Millonarios & $0-1(4-5)$ & Bolognesi & Millonarios \\
\hline 199 & Sul Americana & $04 / 10 / 07$ & Colo Colo & Millonarios & $1-1(6-7)$ & Colo Colo & Millonarios \\
\hline 200 & Sul Americana & $14 / 11 / 07$ & River Plate & Arsenal & $0-0(2-4)$ & Arsenal & Arsenal \\
\hline 201 & Sul Americana & $27 / 08 / 08$ & Sao Paulo & Atletico Paranaense & $0-0(3-4)$ & Sao Paulo & Atletico Paranaense \\
\hline 202 & Sul Americana & $20 / 08 / 08$ & Estudiantes & Independiente & $2-1(5-3)$ & Estudiantes & Estudiantes \\
\hline 203 & Sul Americana & $25 / 08 / 09$ & Coritiba & Vitoria & $2-0(3-5)$ & Vitoria & Vitoria \\
\hline 204 & Sul Americana & $16 / 09 / 09$ & Goias & Atletico Mineiro & $1-1(6-5)$ & Goias & Goias \\
\hline 205 & Sul Americana & $04 / 11 / 09$ & San Lorenzo & River Plate & $0-1(6-7)$ & River Plate & River Plate \\
\hline 206 & Sul Americana & $23 / 09 / 10$ & San Felipe & Guarani & $1-1(8-7)$ & San Felipe & San Felipe \\
\hline 207 & Sul Americana & $08 / 12 / 10$ & Independiente & Goias & $3-1(5-3)$ & Independiente & Independiente \\
\hline 208 & Sul Americana & $22 / 09 / 11$ & Deportivo Cali & Santa Fe & $1-1(5-6)$ & Santa Fe & Santa Fe \\
\hline 209 & Sul Americana & $20 / 10 / 11$ & Universitario & Godoy Cruz & $1-1(3-2)$ & Godoy Cruz & Universitario \\
\hline
\end{tabular}




\section{Continued}

\begin{tabular}{|c|c|c|c|c|c|c|c|}
\hline 210 & Sul Americana & $17 / 11 / 11$ & Libertad & LDU & $1-0(4-5)$ & LDU & LDU \\
\hline 211 & Sul Americana & $23 / 08 / 12$ & Figueirense & Atletico Goianiense & $1-1(2-4)$ & Atletico Goianiense & Atletico Goianiense \\
\hline 212 & Sul Americana & $27 / 08 / 13$ & Coritiba & Vitoria & $1-0(4-3)$ & Vitoria & Coritiba \\
\hline 213 & Sul Americana & $28 / 08 / 13$ & Nautico & Sport & $2-0(1-3)$ & Nautico & Sport \\
\hline 214 & Sul Americana & $24 / 10 / 13$ & Bahia & Atletico Nacional & $1-0(3-4)$ & Bahia & Atletico Nacional \\
\hline 215 & Sul Americana & $26 / 08 / 14$ & Anzoategui & Universidad Catolica & $1-1(4-5)$ & Anzoategui & Universidad Catolica \\
\hline 216 & Sul Americana & $22 / 10 / 14$ & Penarol & Estudiantes & $2-1(1-3)$ & Estudiantes & Estudiantes \\
\hline 217 & Sul Americana & $23 / 10 / 14$ & Deportivo Capiata & Boca Juniors & $0-1(3-4)$ & Boca Juniors & Boca Juniors \\
\hline 218 & Sul Americana & $15 / 10 / 14$ & Goias & Emelec & $1-0(5-6)$ & Goias & Emelec \\
\hline 219 & Sul Americana & $15 / 10 / 14$ & C Vallejo & Bahia & $2-0(7-6)$ & C Vallejo & C Vallejo \\
\hline 220 & Sul Americana & $26 / 11 / 14$ & Sao Paulo & Atletico Nacional & $1-0(1-4)$ & Atletico Nacional & Atletico Nacional \\
\hline 221 & Sul Americana & $19 / 08 / 15$ & Tolima & Carabobo & $0-0(3-1)$ & Tolima & Tolima \\
\hline 222 & Sul Americana & $17 / 09 / 15$ & Juventud & Emelec & $0-0(2-3)$ & Juventud & Emelec \\
\hline 223 & Sul Americana & $01 / 10 / 15$ & Defensor & Lanus & $0-0(5-3)$ & Defensor & Defensor \\
\hline 224 & Sul Americana & $01 / 10 / 15$ & Chapecoense & Libertad & $1-1(5-3)$ & Chapecoense & Chapecoense \\
\hline 225 & Sul Americana & $09 / 12 / 15$ & Santa Fe & Huracan & $0-0(3-1)$ & Huracan & Santa Fe \\
\hline 226 & Sul Americana & $18 / 08 / 16$ & Zamora & Barcelona & $1-1(3-0)$ & Zamora & Zamora \\
\hline 227 & Sul Americana & $16 / 08 / 16$ & Ohiggins & Wanderers & $0-0(4-5)$ & Wanderers & Wanderers \\
\hline 228 & Sul Americana & $18 / 08 / 16$ & Wilstermann & Sol de America & $1-1(4-5)$ & Wilstermann & Sol de America \\
\hline 229 & Sul Americana & $17 / 08 / 16$ & Plaza & Blooming & $1-0(1-4)$ & Blooming & Blooming \\
\hline 230 & Sul Americana & $28 / 09 / 16$ & Junior Barranquilla & Wanderers & $0-0(4-3)$ & Junior Barranquilla & Junior Barranquilla \\
\hline 231 & Sul Americana & $28 / 09 / 16$ & Belgrano & Coritiba & $1-2(3-4)$ & Coritiba & Coritiba \\
\hline 232 & Sul Americana & $28 / 09 / 16$ & Chapecoense & Independiente & $0-0(5-4)$ & Chapecoense & Chapecoense \\
\hline
\end{tabular}

Table 3. Observed quantity of goals scored by a team starting the penalty shoutout, $r$, and the scores of the other team $s$.

\begin{tabular}{|c|c|c|c|c|c|c|c|c|c|c|c|c|c|}
\hline & & & & & & & $r$ & & & & & & \\
\hline & & 0 & 1 & 2 & 3 & 4 & 5 & 6 & 7 & 8 & 9 & 10 & 11 \\
\hline & 0 & - & 0 & 2 & 5 & 0 & 0 & 0 & 0 & 0 & 0 & 0 & 0 \\
\hline & 1 & 0 & - & 1 & 14 & 14 & 0 & 0 & 0 & 0 & 0 & 0 & 0 \\
\hline & 2 & 1 & 1 & - & 5 & 22 & 0 & 0 & 0 & 0 & 0 & 0 & 0 \\
\hline & 3 & 0 & 5 & 11 & - & 14 & 33 & 0 & 0 & 0 & 0 & 0 & 0 \\
\hline & 4 & 0 & 0 & 16 & 24 & - & 16 & 0 & 0 & 0 & 0 & 0 & 0 \\
\hline \multirow[t]{7}{*}{$s$} & 5 & 0 & 0 & 0 & 0 & 20 & - & 7 & 0 & 0 & 0 & 0 & 0 \\
\hline & 6 & 0 & 0 & 0 & 0 & 0 & 8 & - & 3 & 0 & 0 & 0 & 0 \\
\hline & 7 & 0 & 0 & 0 & 0 & 0 & 0 & 2 & - & 2 & 0 & 0 & 0 \\
\hline & 8 & 0 & 0 & 0 & 0 & 0 & 0 & 0 & 2 & - & 1 & 0 & 0 \\
\hline & 9 & 0 & 0 & 0 & 0 & 0 & 0 & 0 & 0 & 1 & - & 0 & 0 \\
\hline & 10 & 0 & 0 & 0 & 0 & 0 & 0 & 0 & 0 & 0 & 1 & - & 0 \\
\hline & 11 & 0 & 0 & 0 & 0 & 0 & 0 & 0 & 0 & 0 & 0 & 1 & - \\
\hline
\end{tabular}


consider the null hypothesis, $H_{0}^{\prime}: \operatorname{Prob}(R-S \leq d)=\operatorname{Prob}(R-S \geq d)$, for every difference $d$. Considering Hollander's test of bivariate symmetry [14] for $H_{0}$, the RFPW test of symmetry [15] for $H_{0}^{\prime}$, and Wilcoxon's signed rank test for $H_{0}^{\prime}$, we found a statistically significant piece of evidence against such null hypotheses with $p$-values $0.0001,0.0013$, and 0.00000003 , respectively. Thus, it pays to start a penalty shootout.

In sum, it does not matter who begins in tennis tiebreak, but it does matter in soccer penalty shootout.

\section{Conclusions}

In a sample of 345 tennis tiebreak situations and 232 soccer penalty kick situations, we found the current $\mathrm{ABAB}$ system of soccer to be biased in that the team going first has a 59.48 percent advantage to end up as the winner. This figure is close to the 60 percent reported by FIFA. The tennis ABBA system does not exhibit the bias in our sample, as in the previous literature. This means FIFA is not wrong in suggesting the penalty shootout should adopt the tennis tiebreak format.

"Football (soccer) is a simple game," former English player Gary Lineker once said. "Twenty-two men chase a ball for 90 minutes and, at the end, the Germans always win," he added. As for penalty shootouts, the Germans are famed for their penalty-spot prowess after winning five shootouts at major finals (have another look at Table 2 and at Ref. [16]). By adopting the tennis tiebreak system, FIFA would then give no grounds for a cold war in football.

\section{References}

[1] BBC Sport (2017) Penalty Shootouts: EFL to Trial “ABBA" Format in 2017-18. http://www.bbc.com/sport/football/40783273

[2] BBC Sport (2017) Uefa: Penalty Shootout Trial Takes Place in Euro Women's Under-17 Semi-Final. http://www.bbc.com/sport/football/39798344

[3] Jordet, G., Hartman, E., Visscher, C. and Lemmink, K.A.P.M. (2007) Kicks from the Penalty Mark in Soccer: The Roles of Stress, Skill, and Fatigue for Kick Outcomes. Journal of Sports Sciences, 25, 121-129. https://doi.org/10.1080/02640410600624020

[4] Jordet, G. and Hartman, E. (2008) Avoidance Motivation and Choking under Pressure in Soccer Penalty Shootouts. Journal of Sport \& Exercise Psychology, 30, 450-457. https://doi.org/10.1123/jsep.30.4.450

[5] Apesteguia, J. and Palacios-Huerta, I. (2010) Psychological Pressure in Competitive Environments: Evidence from a Randomized Natural Experiment. American Economic Review, 100, 2548-2564. https://doi.org/10.1257/aer.100.5.2548

[6] Palacios-Huerta, I. (2012) Tournaments, Fairness and the Prouhet-Thue-Morse Sequence. Economic Inquiry, 50, 848-849.

https://doi.org/10.1111/j.1465-7295.2011.00435.x

[7] Palacios-Huerta, I. (2014) Beautiful Game Theory: How Soccer Can Help Economics. Princeton University Press, Princeton. https://doi.org/10.1515/9781400850310

[8] Anbarci, N., Sun, C.J. and Unver, M. (2015) Designing Fair Tiebreak Mechanisms: The Case of FIFA Penalty Shootouts. Boston College Working Papers in Economics, 
No. 871. https://dlib.bc.edu/islandora/object/bc-ir:104596/datastream/PDF/view

[9] Brams, S.J. and Ismail, M.S. (2018) Making the Rules of Sports Fairer. SIAM Review, 60, 181-202. https://doi.org/10.1137/16M1074540

[10] Vandebroek, T.P., McCann, B.T. and Vroom, G. (2018) Modeling the Effects of Psychological Pressure on First-Mover Advantage in Competitive Interactions: The Case of Penalty Shoot-Outs. Journal of Sports Economics. (In press) https://doi.org/10.1177/1527002516672060

[11] Echenique, F. and Rodriguez, J.L. (2017) Abab or Abba? The Arithmetics of Penalty Shootouts in Soccer. http://people.hss.caltech.edu/ fede/wp/penales.pdf

[12] Cohen-Zada, D., Krumer, A. and Shapir, O.F. (2017) Take a Chance on ABBA. IZA Institute of Labor Economics Discussion Papers, No. 10878. http://ftp.iza.org/dp10878.pdf

[13] Cohen-Zada, D., Krumer, A. and Shapir, O.F. (2018) Testing the Effect of Serve Order in Tennis Tiebreak. Journal of Economic Behavior \& Organization, 146, 106-115. https://doi.org/10.1016/j.jebo.2017.12.012

[14] Hollander, M. (1971) A Nonparametric Test for Bivariate Symmetry. Biometrika, 58, 203-212. https://doi.org/10.1093/biomet/58.1.203

[15] Randles, R.H., Fligner, M.A., Policello, II G.E. and Wolfe, D.A. (1980) An Asymptotically Distribution-Free Test for Symmetry versus Asymmetry. Journal of the American Statistical Association, 75, 168-172. https://doi.org/10.1080/01621459.1980.10477448

[16] Lineker, G. (2017) What Makes the Perfect World Cup Shootout Penalty? http://www.bbc.co.uk/guides/zgg334j\#z22qq6f 